\title{
Component Analysis of Unsteady Hydrodynamic Force of Closed-Type Centrifugal Pump with Single Blades of Different Blade Outlet Angles
}

\author{
Yasuyuki Nishi $^{1}$ and Junichiro Fukutomi ${ }^{2}$ \\ ${ }^{1}$ Department of Mechanical Engineering, Ibaraki University, 4-12-1 Nakanarusawa-cho, Hitachi-shi, Ibaraki 316-8511, Japan \\ ${ }^{2}$ Institute of Technology and Science, The University of Tokushima, 2-1 Minamijosanjima-cho, Tokushima-shi, \\ Tokushima 770-8506, Japan \\ Correspondence should be addressed to Yasuyuki Nishi; y-nishi@mx.ibaraki.ac.jp
}

Received 16 March 2015; Accepted 8 July 2015

Academic Editor: Robert C. Hendricks

Copyright (C) 2015 Y. Nishi and J. Fukutomi. This is an open access article distributed under the Creative Commons Attribution License, which permits unrestricted use, distribution, and reproduction in any medium, provided the original work is properly cited.

\begin{abstract}
Single-blade centrifugal impellers for sewage systems undergo both unsteady radial and axial thrusts. Therefore, it is extremely important for the improvement of pump reliability to quantitatively grasp these fluctuating hydrodynamic forces and determine the generation mechanism behind them. In this study, we conducted component analyses of radial and axial thrusts of closed, single-blade centrifugal pumps with different blade outlet angles by numerical analysis while considering leakage flow. The results revealed the effect of the blade outlet angle on the components of radial and axial thrusts. For increased flow rates, the time-averaged values of the pressure component were similar for all impellers, although its fluctuating components were higher for impellers with larger blade outlet angles. Moreover, the fluctuating inertia component of the impeller with a blade outlet angle of $8^{\circ}$ decreased as the flow rate increased, whereas those with $16^{\circ}$ and $24^{\circ}$ angles increased. Therefore, the radial thrust on the hydraulic part was significantly higher for impellers with high blade outlet angles.
\end{abstract}

\section{Introduction}

Sewage pumps for transporting fluids with solid and fibrous foreign bodies are required to have a high foreign body passage diameter; thus, a centrifugal pump with a single wide blade is often used. Currently, foreign body passage is evaluated according to the passed particle size compared to the pump diameter [1]. Nevertheless, even for centrifugal pumps with many blades, geometrical manufacturing errors cause disproportional fluid force [2]. The single-blade centrifugal pumps in current usage are equipped with an axially nonsymmetric volute casing around an axially nonsymmetric impeller. For this reason, the static pressure distribution surrounding the impeller is extremely nonuniform, and an excessively fluctuating radial thrust acts on the impeller $[3,4]$. Excessive radial thrust shortens the lifetime of the bearing and shaft seals and lessens the pump's reliability by causing shaft vibration; thus, it is essential to determine the development mechanism of such vibration.

Regarding the radial thrust in single-blade centrifugal pumps, such subjects have been investigated as the effect of the number of blades $[3,5]$, winding angle of the blade [3], distribution of the blade angle [6] and its relationship with the static pressure between the blades [4], effect of the casing shape $[3,6]$, effectiveness of adding extra mass $[4,7]$, and relationship to vibration of the casing [7]. In addition, the relationship between the hydrodynamic forces acting on the impeller and the whirling of the impeller has been investigated $[5,8-10]$. We have also proposed a design method for single-blade centrifugal impellers and have discovered, for a closed-type centrifugal impeller with a single blade that was designed using this design method as a basis, how the performance characteristics relate to the radial thrust [11]. While modeling the components of the radial 
TABLE 1: Specifications of test impellers.

\begin{tabular}{lc}
\hline Impeller suction diameter $D_{0}$ & $67 \mathrm{~mm}$ \\
Impeller inner diameter $D_{1}$ & $58 \mathrm{~mm}$ \\
Impeller outer diameter $D_{2}$ & $194 \mathrm{~mm}$ \\
Blade inlet width $b_{1}$ & $56 \mathrm{~mm}$ \\
Blade outlet width $b_{2}$ & $56 \mathrm{~mm}$ \\
Blade inlet angle $\beta_{b_{1}}$ & $13^{\circ}$ \\
Blade outlet angle $\beta_{b_{2}}$ & $16^{\circ}$ \\
\hline
\end{tabular}

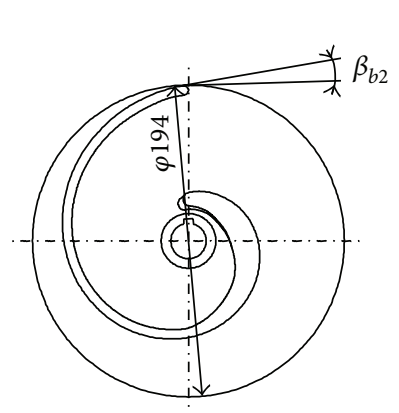

Section A-A

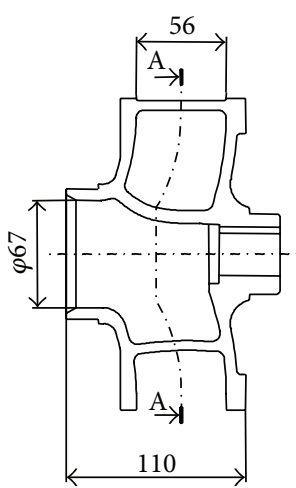

Figure 1: Test impeller. thrust using an unsteady conservation-of-momentum law to illustrate their behavior [12], we also explained how they were affected by the blade outlet angle [13]. However, hardly any research has been made on the axial thrust. In our previous studies, the hydrodynamic forces acting on the rear and front shroud parts were neglected in the numerical analyses. For this purpose, we carried out numerical analyses with consideration for leakage flows behind the rear and front shrouds and revealed the behaviors of radial and axial thrusts [14]. We also examined the effect of the blade outlet angel on the radial and axial thrusts [15].

As a continuation of a previous report [15], we conducted numerical analyses herein with consideration of leakage flows behind the rear and front shrouds for closed, single-blade centrifugal pumps with different blade outlet angles. We conducted component analyses of radial and axial thrusts and revealed the effect of the blade outlet angle on the thrust components.

\section{Test Pumps}

The three closed, single-blade centrifugal impellers used as test pumps for this study had blade outlet angles $\beta_{b 2}$ of $8^{\circ}$, $16^{\circ}$, and $24^{\circ}$, respectively [15]. An overview of the test impeller $\left(\beta_{b 2}=8\right)$ is shown in Figure 1 , and its specifications are given in Table 1 . An overview of the test pump is also given in Figure 2. The impeller's rotational speed was $1740 \mathrm{~min}^{-1}$. The impeller with $\beta_{b 2}=8$ had a passed particle size of $56 \mathrm{~mm}$ and had been designed on the basis of the design method reported previously [11]. This design method stipulates the passed particle size beforehand, and the impeller design is implemented using as design parameters, first, the relative velocity ratio of the blade inlet and blade outlet and, second,

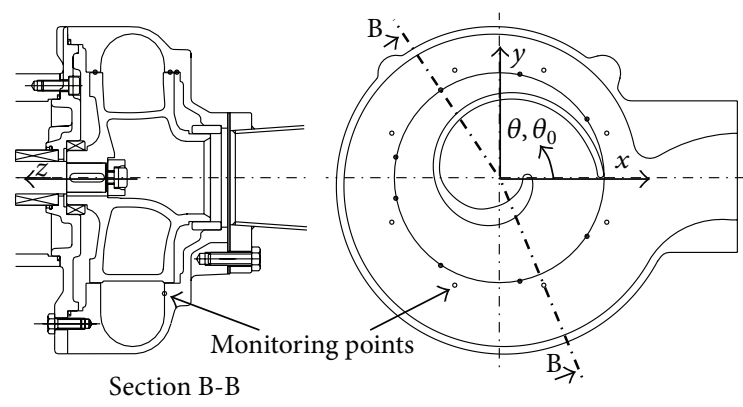

Figure 2: Test pump.

the absolute velocity ratio of the impeller suction inlet and the blade inlet. The impellers with $\beta_{b 2}=16$ and $\beta_{b 2}=24$ have meridian planes that are equivalent to that for the impeller with $\beta_{b 2}=8$, and, as shown in Table 1, only the blade outlet angle is varied. This pump has three narrow gaps between the impeller and the casing, as seen in Figure 2. These are the liner-ring gap (the gap with the casing wearing ring), the gap with the rear shroud part, and the gap with the front shroud part. The gap widths are, respectively, 0.75, 0.5 , and $0.5 \mathrm{~mm}$ and the corresponding gap lengths are $17,10.1$, and $10.5 \mathrm{~mm}$. Moreover, no leakage flow from the pumps at the rear shroud side was noted.

The coordinate system is defined by the $x_{-}, y$-, and $z$ axes, as shown in Figure 2. The blade phase angle $\theta_{0}$ is taken in the anticlockwise direction from the $x$-axis, and the point where the end of the blade outlet passes through the $x$-axis is assumed as $\theta_{0}=0^{\circ}$ (the position shown in Figure 2). Eight monitoring points of the static pressure were provided on the wall surface on the side of the front shroud part at positions $10 \mathrm{~mm}$ radially outwards from the impeller outlet in an anticlockwise direction from the $x$-axis as $\theta=0^{\circ}$.

\section{Numerical Analysis Method}

For the numerical analysis, we performed a three-dimensional unsteady-flow analysis using the general-purpose thermofluid analysis code ANSYS CFX13.0 [15]. The governing equations include a continuous equation [16] and ReynoldsAveraged Navier-Stokes equations [16], and a SST (Shear Stress Transport) [16] was used for the turbulence model.

The computational domain and computational grid for the pump part are shown, respectively, in Figures 3(a) and 3 (b). This study differs from past research [11-13] in that the gaps between the outer surfaces of the rear and front shroud parts of the impeller and the casing are actually modeled. The computation domain consists of the suction pipe, impeller, volute casing, regions in the gaps with the rear and front shroud parts, and discharge pipe. The computational grids for the impellers with $\beta_{b 2}=8, \beta_{b 2}=16$, and $\beta_{b 2}=24$ have 690,000, 705,000, and 707,000 elements, respectively, where the total number of elements in the grids is $2,328,000,2,343,000$, and 2,345,000, respectively. Here, the computational grids for the suction pipe, volute casing, regions in the gaps with the rear and front shroud parts, and the discharge pipe are identical in all cases, with, respectively, 


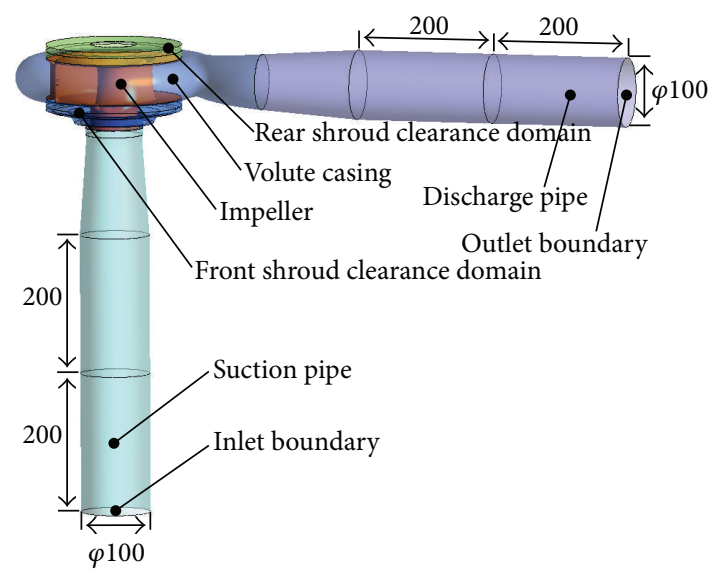

(a) Computational domain

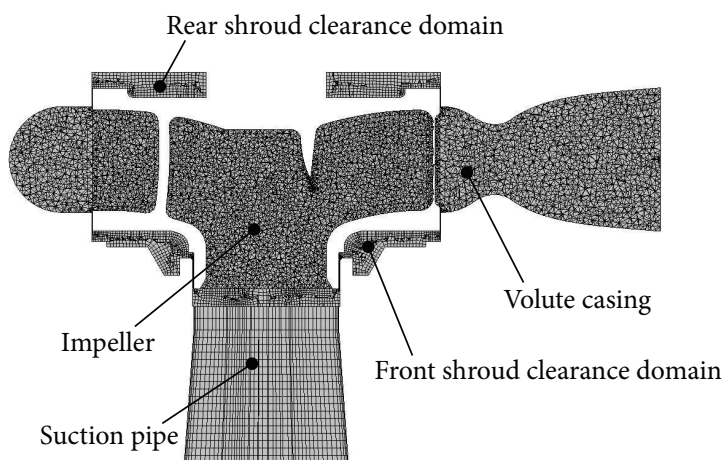

(b) Computational grids of pump part

FIGURE 3: Computational model.

$149,000,462,000,340,000,572,000$, and 115,000 elements. The number of elements in the gaps counted radially is five for the liner-ring gap and four for both the rear shroud part and front shroud part gaps. A nonstructured tetrahedral grid is used as the computational grid for the impeller and volute casing, whereas a nonstructured hexahedral grid is used for the others.

To study the dependence of the computational model on the grid used, we performed analyses for the $\beta_{b 2}=8$ case, where there were, respectively, 8,6 , and 6 elements in the radial direction in the liner-ring gap, rear shroud part gap, and front shroud part gap, and computational grids for the rear shroud part gap and front shroud part gap regions had 561,000 and 1,017,000 elements, respectively, with a total grid number of approximately 2,994,000 elements. The results showed that the differences between the two meshes in the flow rate, at the maximum efficiency point in the analysis results for the $\beta_{b 2}=8$ case, were $0.2 \%$ for the head, $0.3 \%$ for the shaft power, $0.7 \%$ for the root-mean-square value of the total radial thrust, and $4.1 \%$ for the time-averaged value of the total axial thrust. For an impeller with $\beta_{b 2}=8$, we performed analyses with volute casing computational grid numbers of $1,035,000$ and 693,000 elements, with a total grid number of approximately 2,904,000 elements. The results showed that the differences between the two meshes in the flow rate, at the maximum efficiency point in the analysis results for the

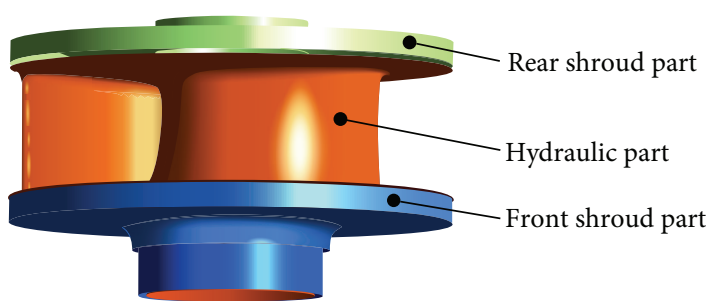

FIgURE 4: Impeller surfaces.

$\beta_{b 2}=8$ case, were $1.8 \%$ for the head, $1.6 \%$ for the shaft power, $0.6 \%$ for the root-mean-square value of the total radial thrust, and $3.7 \%$ for the time-averaged value of the total axial thrust. As mentioned above, we verified that the grid dependence for this computational model was comparatively small [14].

The boundary conditions for the suction pipe inlet were set as those on the inlet boundary, and for the discharge pipe as those on the outlet boundary, and were given as the mass flow rate on the inlet boundary, a static pressure (gauge pressure) on the outlet boundary of $0 \mathrm{~Pa}$, and the rotational speed ( $n=1740 \mathrm{~min}^{-1}$ ) in the impeller domain. The wall surface was assumed to be a nonslip condition, and the boundary between the rotating and static domains was conjugated using the transient rotor-stator technique [17]. The time step was set such that 360 steps were a single rotation of the impeller, and the calculations were continued until the flow became nearly periodic.

\section{Modeling of the Components of Radial and Axial Thrusts}

To understand the behavior of unsteady hydrodynamic forces on each part of the impellers, we divided the impeller surface into three parts, as shown in Figure 4. We classified the blade surface and the rear and front shroud inner surfaces as the hydraulic part, the rear shroud outer surface, including the outer circumference surface, as the rear shroud part, and the front shroud outer surface, including the front shroud circumference surface, as the front shroud part. The total radial thrust $F_{r}$ and the total axial thrust $F_{a}$ applied on the impeller are expressed as follows [14]:

$$
\begin{aligned}
& F_{r}=F_{r H}+F_{r R}+F_{r F} \\
& F_{a}=F_{a H}+F_{a R}+F_{a F} .
\end{aligned}
$$

Here, $F_{r H}$ is the radial thrust on the hydraulic part [N], $F_{r R}$ is the radial thrust on the rear shroud part [N], $F_{r F}$ is the radial thrust on the front shroud part $[\mathrm{N}], F_{a H}$ is the axial thrust on the hydraulic part [N], $F_{a R}$ is the axial thrust on the rear shroud part [N], and $F_{a F}$ is the axial thrust on the front shroud part [N]. For the radial and axial thrusts on the hydraulic part, we modeled the components to examine their behaviors in detail. $F_{r H}$ and $F_{a H}$ are expressed as follows:

$$
\begin{aligned}
& F_{r H}=\sqrt{F_{H x}^{2}+F_{H y}^{2}} \\
& F_{a H}=F_{H z} .
\end{aligned}
$$


Let the fluid domain inside the impeller be the test volume, let the impeller suction inlet be the test inlet, and let the impeller outlet be the test outlet. Applying the unsteady momentum conservation law, the $x, y$, and $z$ components of the hydrodynamic force on the hydraulic part inside the impeller $F_{H}$ can be expressed as follows [14]:

$$
\begin{aligned}
& F_{H x}=F_{I x}+F_{M x}+F_{P x}+F_{\tau x} \\
& F_{H y}=F_{I y}+F_{M y}+F_{P y}+F_{\tau y} \\
& F_{H z}=F_{I z}+F_{M z}+F_{P z}+F_{\tau z} .
\end{aligned}
$$

Here, $F_{I}$ is the inertia component [N], $F_{M}$ is the momentum component [N], $F_{P}$ is the pressure component [N], and $F_{\tau}$ is the shear stress component $[\mathrm{N}]$.

The inertia component $F_{I}$ is caused by the time-varying momentum of the fluid inside the impeller. Its $x, y$, and $z$ components can be obtained as follows:

$$
\begin{aligned}
& F_{I x}=-\rho \int_{V} \frac{d v_{x}}{d t} d V \\
& F_{I y}=-\rho \int_{V} \frac{d v_{y}}{d t} d V \\
& F_{I z}=-\rho \int_{V} \frac{d v_{z}}{d t} d V .
\end{aligned}
$$

Here, $V$ is the test volume, which is the fluid domain inside the impeller.

The momentum component $F_{M}$ is caused by the difference between the fluid momentum at the impeller outlet and inlet. Its $x, y$, and $z$ components can be obtained as follows:

$$
\begin{aligned}
& F_{M x}=-\rho\left(\int_{A_{2}} v_{x 2} v_{r 2} d A-\int_{A_{0}} v_{x 0} v_{z 0} d A\right) \\
& F_{M y}=-\rho\left(\int_{A_{2}} v_{y 2} v_{r 2} d A-\int_{A_{0}} v_{y 0} v_{z 0} d A\right) \\
& F_{M z}=-\rho\left(\int_{A_{2}} v_{z 2} v_{r 2} d A-\int_{A_{0}} v_{z 0} v_{z 0} d A\right) .
\end{aligned}
$$

Here, $A_{2}$ is the impeller outlet area $\left[\mathrm{m}^{2}\right]$ and $A_{0}$ is the area of the impeller suction inlet $\left[\mathrm{m}^{2}\right]$.

The pressure component $F_{P}$ is caused by the difference between the fluid momentum at the impeller outlet and inlet. Its $x, y$, and $z$ components can be obtained as follows:

$$
\begin{aligned}
& F_{P x}=-\int_{A_{2}}\left(P_{2}-P_{s}\right) \cos \theta d A \\
& F_{P y}=-\int_{A_{2}}\left(P_{2}-P_{s}\right) \sin \theta d A \\
& F_{P z}=-\int_{A_{0}}\left(P_{0}-P_{s}\right) d A .
\end{aligned}
$$

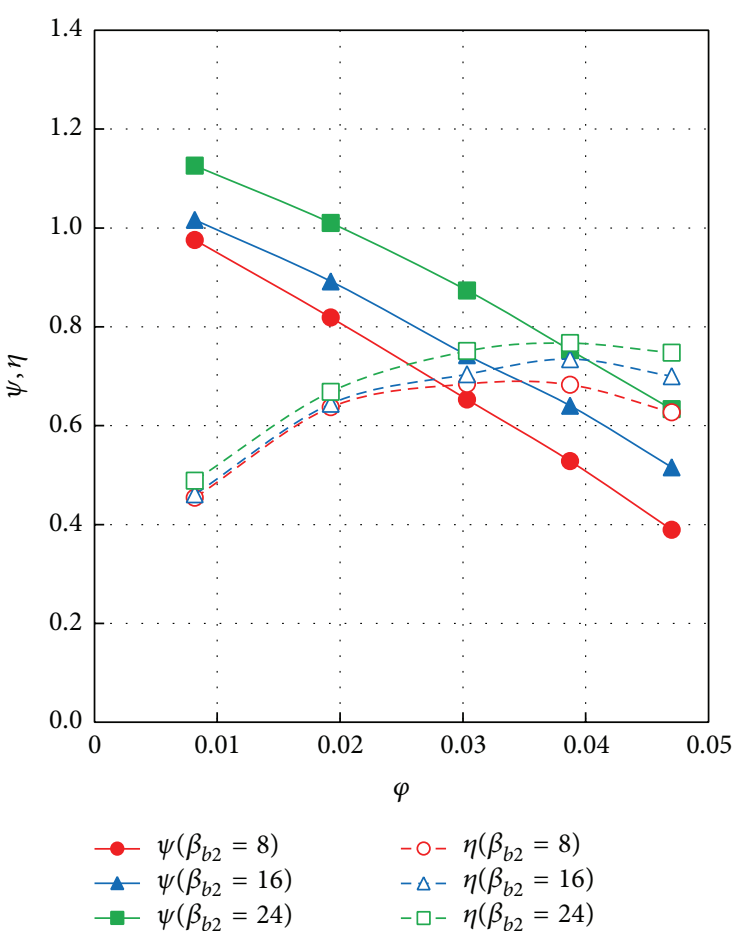

FIGURE 5: Performance curves.

The shear stress component $F_{\tau}$ is applied on the fluid surface at the impeller outlet and at the suction inlet. Its $x$, $y$, and $z$ components can be obtained as follows:

$$
\begin{aligned}
& F_{\tau x}=-\left(\int_{A_{2}} \tau_{2} \sin \gamma \sin \theta d A+\int_{A_{0}} \tau_{0} \cos \theta d A\right) \\
& F_{\tau y}=-\left(\int_{A_{2}} \tau_{2} \sin \gamma \cos \theta d A+\int_{A_{0}} \tau_{0} \sin \theta d A\right) \\
& F_{\tau z}=-\left(\int_{A_{2}} \tau_{2} \cos \gamma d A\right) .
\end{aligned}
$$

Here, $\gamma$ is the angle between the $z$-axis and the direction of the shear stress on the impeller outlet surface.

\section{Analysis Results and Considerations}

5.1. Comparisons of Pump Characteristics. Figure 5 shows the comparisons of the pump characteristics for $\beta_{b 2}=8$, $\beta_{b 2}=16$, and $\beta_{b 2}=24$. Moreover, it has been confirmed that the calculated values of the head coefficient $\psi$ and the pump efficiency $\eta$ qualitatively match the experimental results [15]. As the flow rate increased, the head coefficient $\psi$ was higher for impellers with larger $\beta_{b 2}$. For the flow rate at the maximum efficiency point $\varphi=0.030$ for $\beta_{b 2}=8$, values of $\psi$ for $\beta_{b 2}=16$ and 24 were larger than that of $\beta_{b 2}=8$ by $13.7 \%$ and $33.8 \%$, respectively. This result is presumably due to the increase in the circumferential component of the absolute velocity at the blade outlet with greater blade outlet angles. For the pump efficiency $\eta$, the flow rate with maximum 


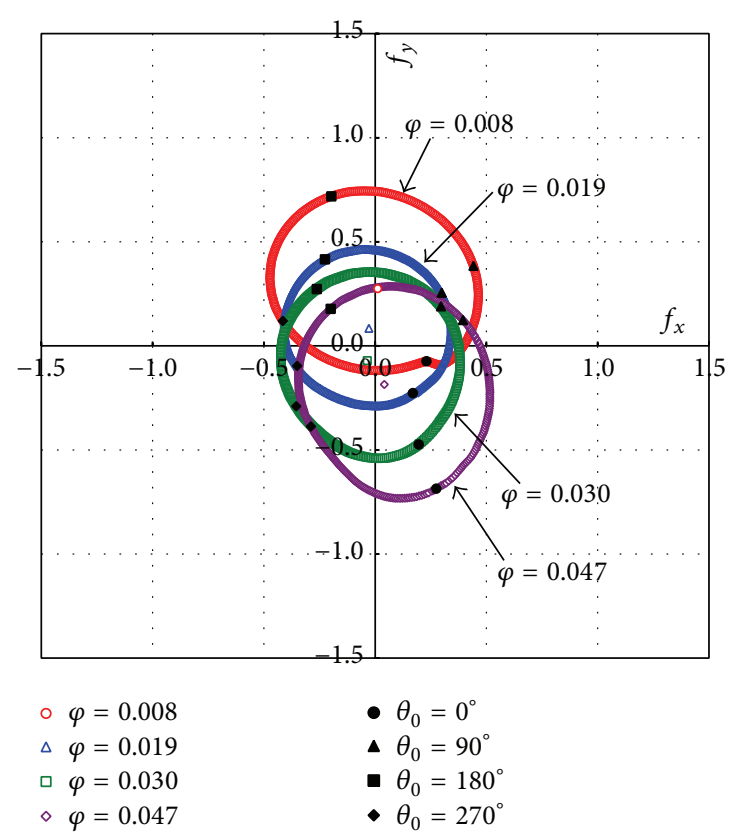

(a) Total

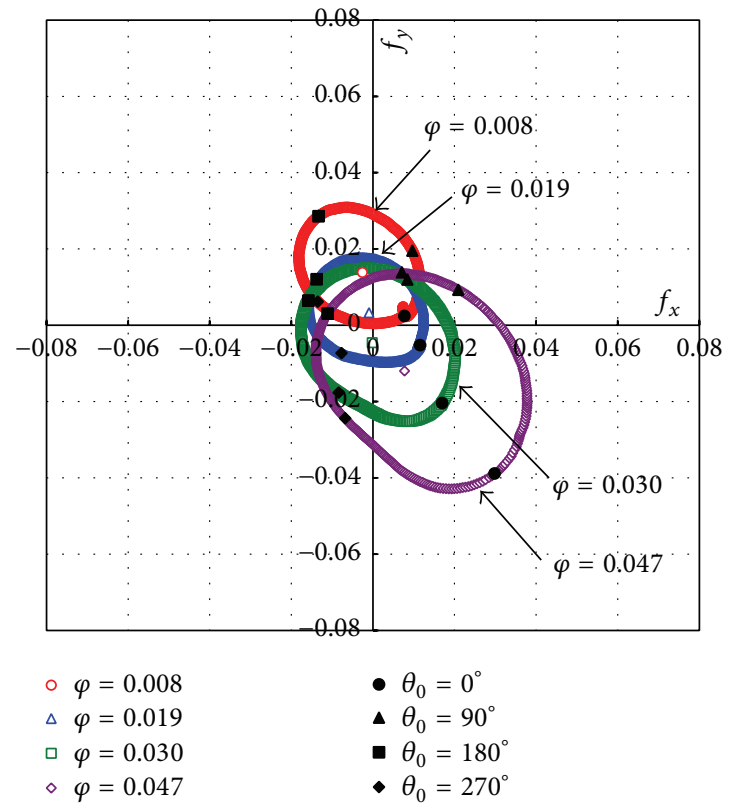

(c) Rear shroud part

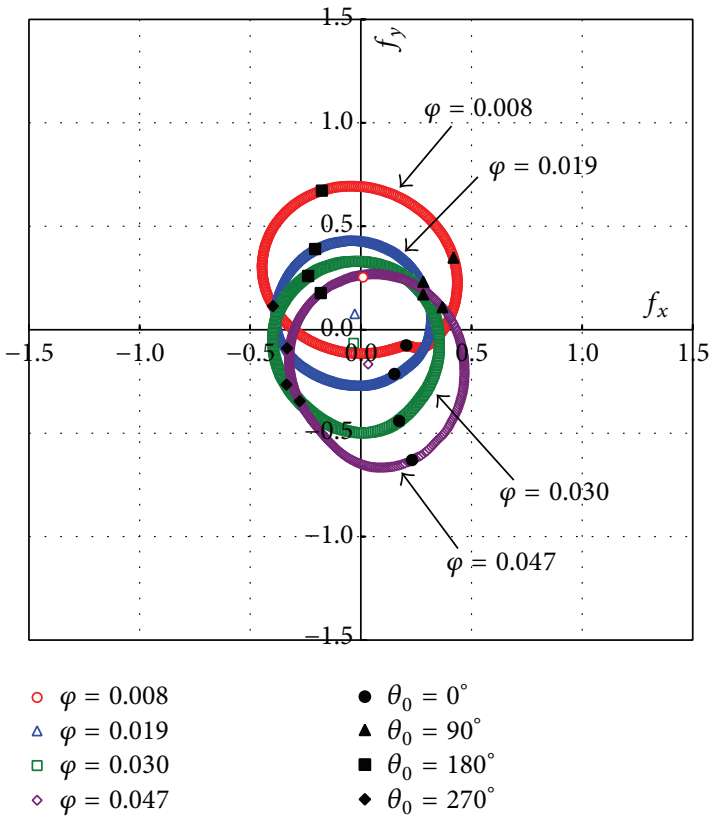

(b) Hydraulic part

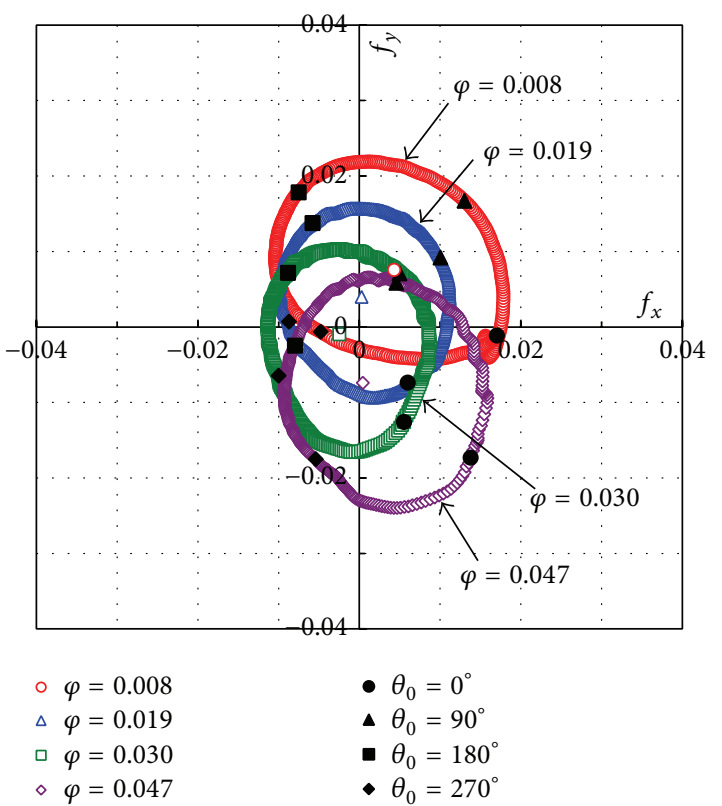

(d) Front shroud part

FIGURE 6: Lissajous figures of radial thrust coefficient on each part $\left(\beta_{b 2}=8\right)$.

efficiency was $\varphi=0.030$ for $\beta_{b 2}=8$, whereas it moved to a higher rate of $\varphi=0.039$ for $\beta_{b 2}=16$ and 24. At $\varphi=0.039$, $\eta$ was observed to be 0.735 and 0.767 for $\beta_{b 2}=16$ and 24 , respectively, indicating that higher $\eta$ values occurred for impellers with higher $\beta_{b 2}$ values. The flow rate at the point of maximum efficiency is thought to be moving toward a larger flow rate because the theoretical pump head for a finite number of blades increases as the blade outlet angle increases, and the matching point with Worster's characteristic formula for the volute [18] then moves toward a larger flow rate.
5.2. Radial Thrust and Comparison of Its Components. The radial thrust $F_{r}$ can be defined by the following formula as the vector sum of the time-averaged value (an average component) and a fluctuating component $[3,4,19]$ :

$$
F_{r}=\bar{F}_{r}+\Delta F_{r} .
$$

Here, $\bar{F}_{r}$ is the time-averaged value and $\Delta F_{r}$ is the fluctuating component.

Figures 6(a)-6(d) and 7(a)-7(d) show the Lissajous figures of the total, hydraulic part, rear shroud part, and front 


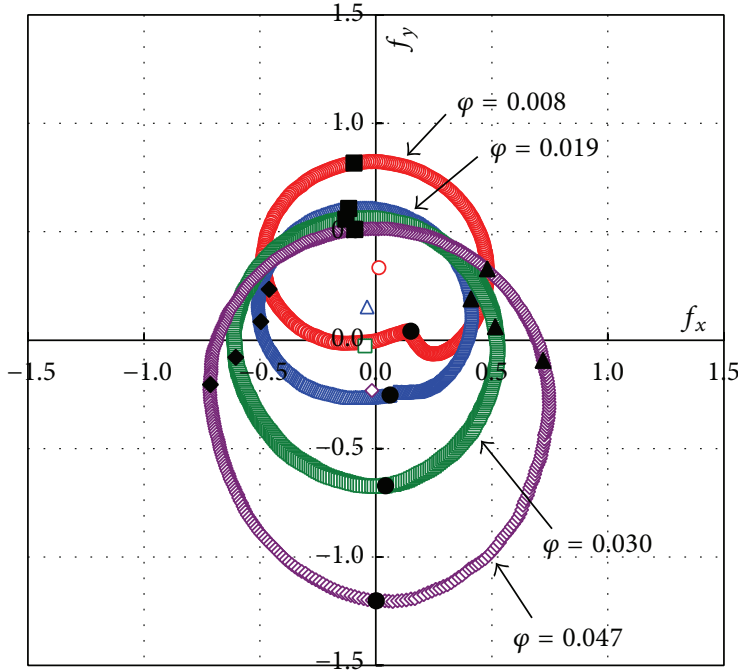
○ $\varphi=0.008$
$\Delta \varphi=0.019$
ㅁ $\varphi=0.030$
$\diamond \varphi=0.047$
- $\theta_{0}=0^{\circ}$
\ $\theta_{0}=90^{\circ}$
- $\theta_{0}=180^{\circ}$
- $\theta_{0}=270^{\circ}$

(a) Total

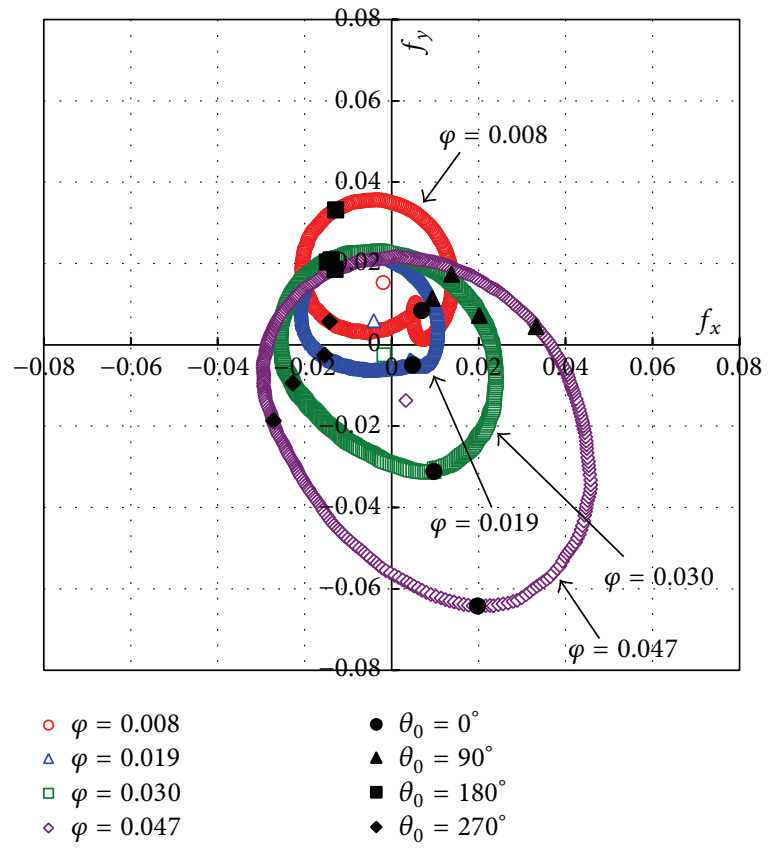

(c) Rear shroud part

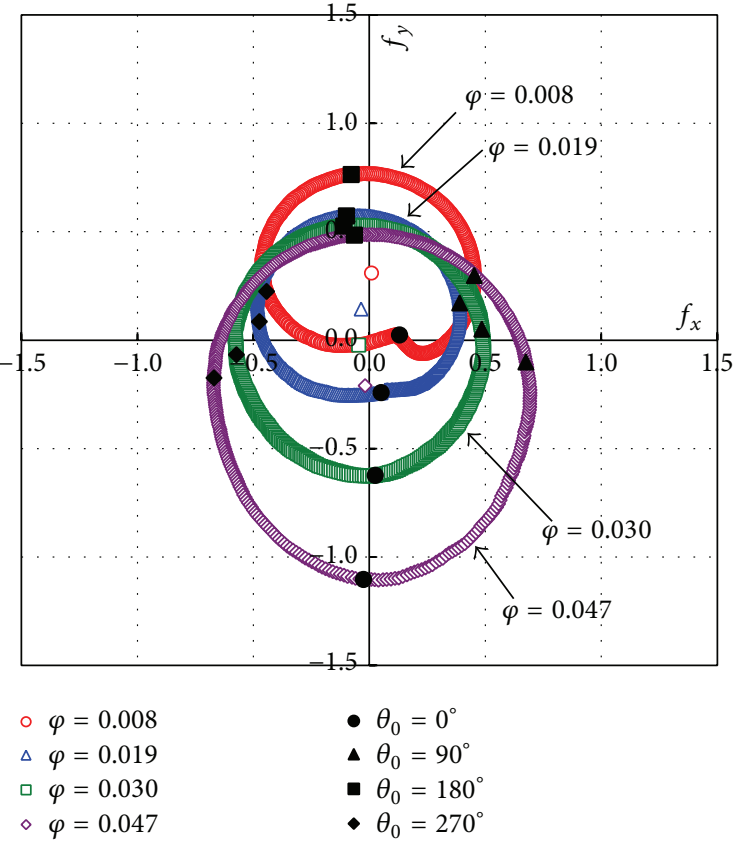

(b) Hydraulic part

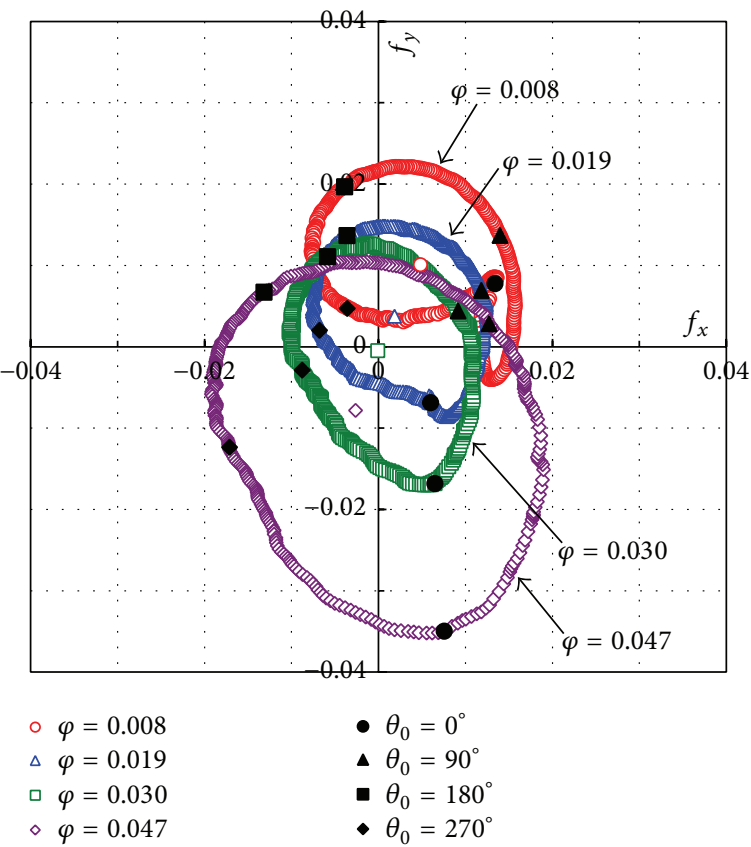

(d) Front shroud part

FIGURE 7: Lissajous figures of radial thrust coefficient on each part $\left(\beta_{b 2}=24\right)$.

shroud part with the radial thrust coefficient. Here, each radial thrust was obtained by integrating the pressure and the shear stress on the wall of each impeller part. For each radial thrust coefficient, the values at $\theta_{0}=0,90,180$, and 270 are shown, respectively, by $\bullet, \mathbf{\Lambda}, \mathbf{\square}$, and $\bullet$, and the timeaveraged values are given using the same symbols as those for the radial thrust coefficient. These are represented similarly in Figures 9-11. The time-averaged values $\bar{f}_{r}$ of the radial thrust coefficient and the root-mean-square values of the fluctuating component $\Delta f_{r}$ for the total, hydraulic part, rear shroud part, and front shroud part are shown in Figures 8(a)$8(\mathrm{~d})$. The difference between the calculated and experimental values for the time-averaged values $\bar{f}_{r}$ of the radial thrust coefficient and the root-mean-square values of the fluctuating component $\Delta f_{r}$ (where $\beta_{b 2}=8$ ) was found to be $15.4 \%$ and $1.8 \%$ at $\varphi=0.008$, respectively. Moreover, at $\varphi=0.047$, it 

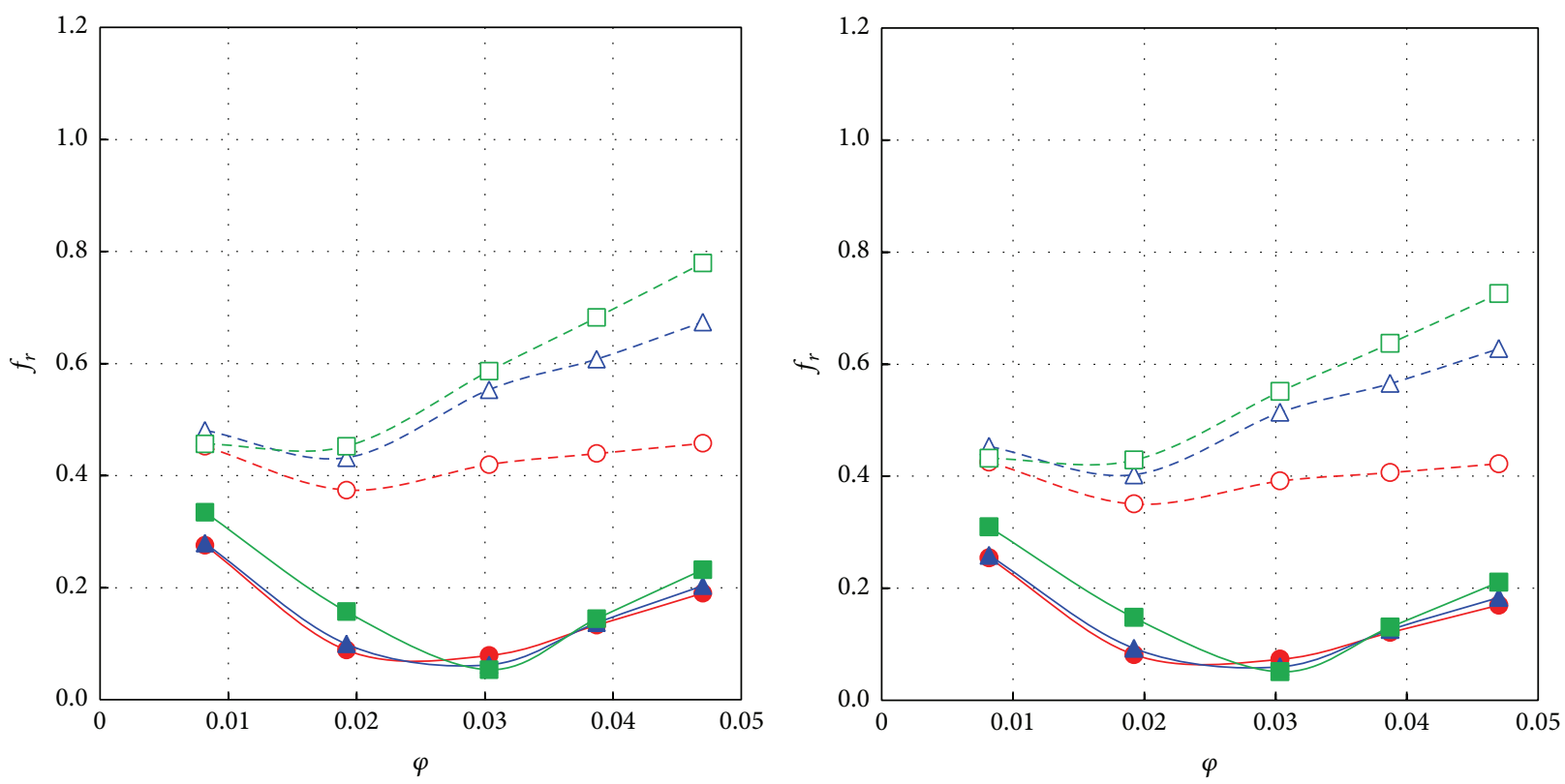

$\rightarrow$ Time-averaged $f\left(\beta_{b 2}=8\right)$

$\triangle$ Time-averaged $f\left(\beta_{b 2}=16\right)$

$\multimap$ Time-averaged $f\left(\beta_{b 2}=8\right)$

$\rightarrow$ Time-averaged $f\left(\beta_{b 2}=16\right)$

$\rightarrow$ Time-averaged $f\left(\beta_{b 2}=24\right)$

-o- Root-mean-square $\Delta f\left(\beta_{b 2}=8\right)$

$-\Delta$ - Root-mean-square $\Delta f\left(\beta_{b 2}=16\right)$

- - - Root-mean-square $\Delta f\left(\beta_{b 2}=24\right)$

(a) Total

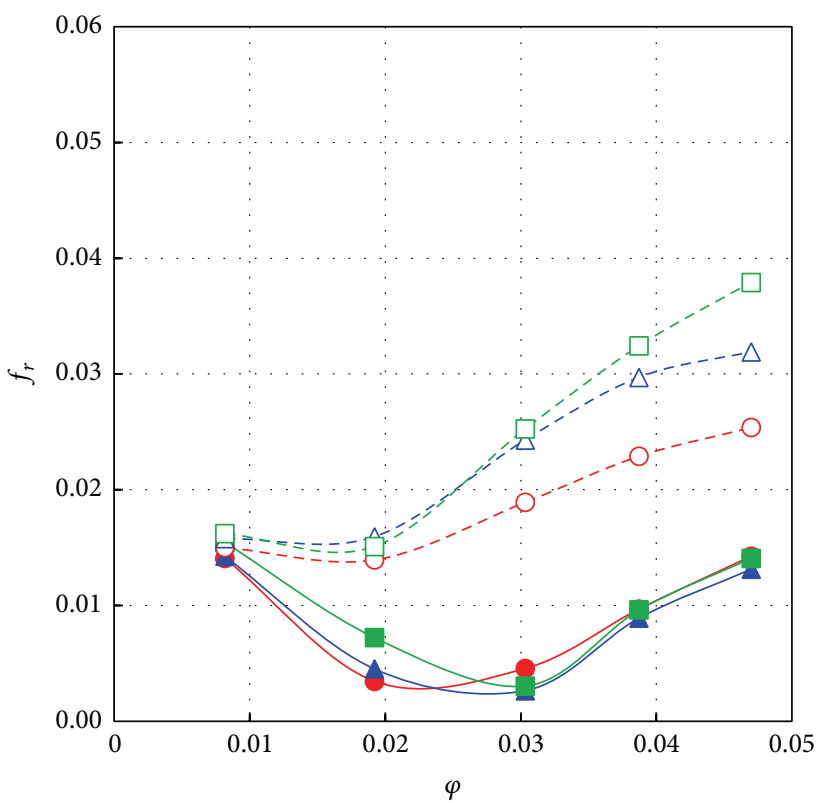

$\rightarrow$ Time-averaged $f\left(\beta_{b 2}=24\right)$

- o- Root-mean-square $\Delta f\left(\beta_{b 2}=8\right)$

- $\Delta$ - Root-mean-square $\Delta f\left(\beta_{b 2}=16\right)$

- - Root-mean-square $\Delta f\left(\beta_{b 2}=24\right)$

(b) Hydraulic part

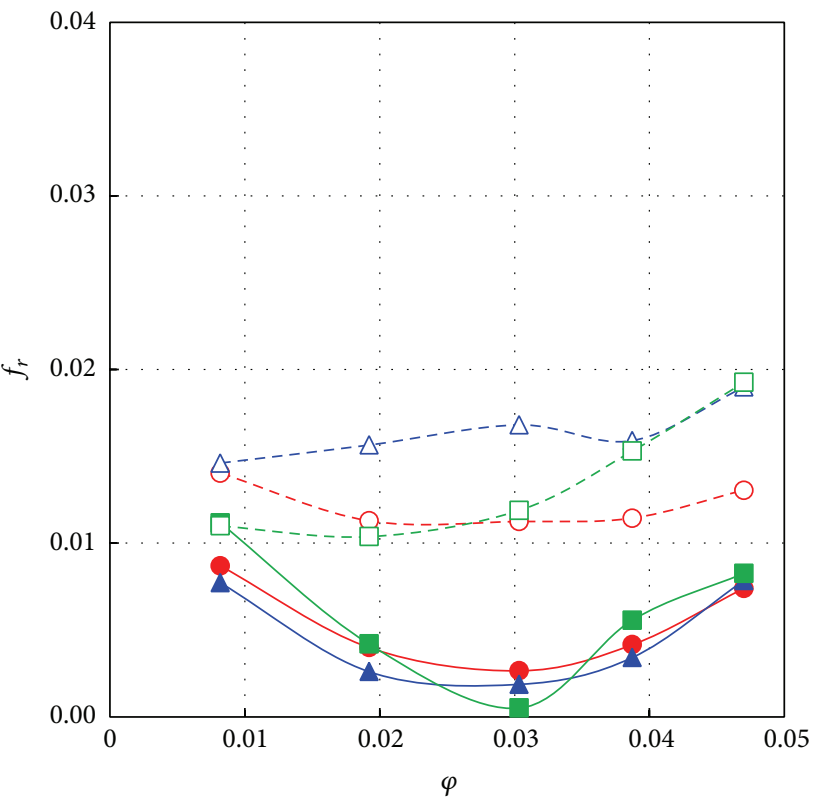

$\rightarrow$ Time-averaged $f\left(\beta_{b 2}=8\right)$

$\simeq$ Time-averaged $f\left(\beta_{b 2}=16\right)$

$\rightarrow$ Time-averaged $f\left(\beta_{b 2}=24\right)$

-o- Root-mean-square $\Delta f\left(\beta_{b 2}=8\right)$

- $\Delta$ - Root-mean-square $\Delta f\left(\beta_{b 2}=16\right)$

- - Root-mean-square $\Delta f\left(\beta_{b 2}=24\right)$

(c) Rear shroud part

$\rightarrow$ Time-averaged $f\left(\beta_{b 2}=8\right)$
$\longleftarrow$ Time-averaged $f\left(\beta_{b 2}=16\right)$
$\because-$ Time-averaged $f\left(\beta_{b 2}=24\right)$
$-\circ$ - Root-mean-square $\Delta f\left(\beta_{b 2}=8\right)$
$-\Delta-$ Root-mean-square $\Delta f\left(\beta_{b 2}=16\right)$
$-\square-$ Root-mean-square $\Delta f\left(\beta_{b 2}=24\right)$

(d) Front shroud part

FIGURE 8: Radial thrust coefficient $\left(f_{r}\right)$ on each part as functions of flow rate coefficient $(\varphi)$. 


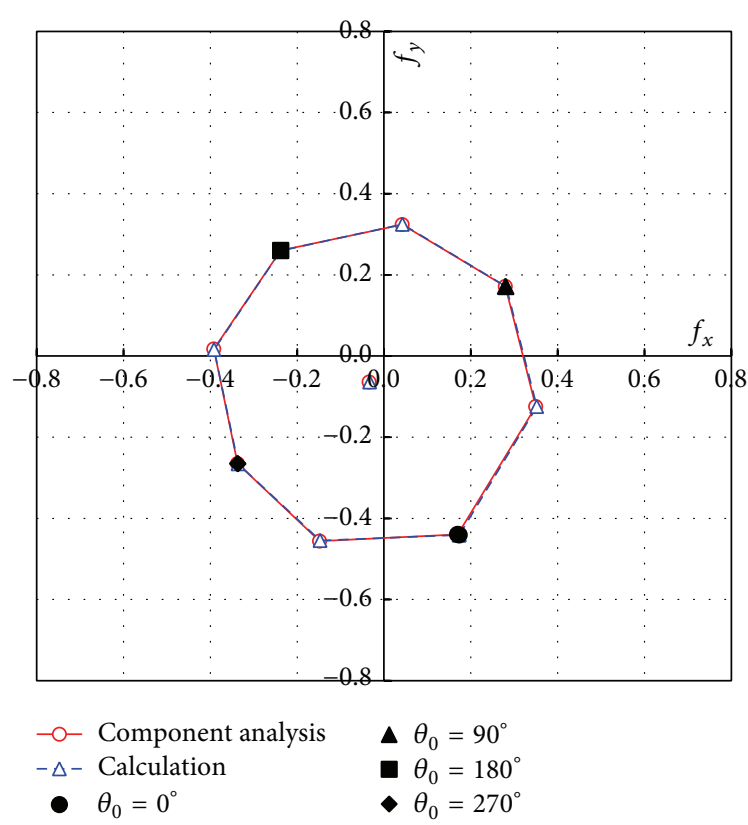

(a) $\beta_{b 2}=8$

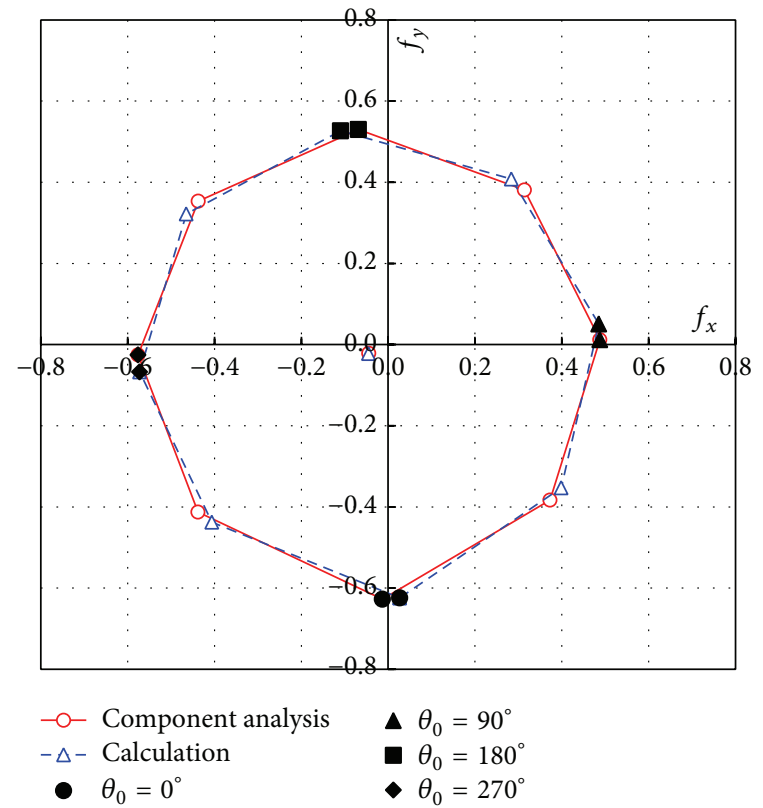

(b) $\beta_{b 2}=24$

FIGURE 9: Lissajous figures of hydraulic part radial thrust coefficient $(\varphi=0.030)$.

was $-24.4 \%$ and $-9.7 \%$, respectively. However, we verified that the experimental results qualitatively correlated with the calculated results for the time-averaged value $\bar{f}_{r}$ of radial thrust coefficient and the root-mean-square values of the fluctuating component $\Delta f_{r}[15]$.

For $\beta_{b 2}=8$ and 24, the Lissajous figures of the total radial thrust coefficient moved to the $+y$ and $-y$ directions for decreased and increased flow rates, respectively. The Lissajous figure of the radial thrust coefficient for each part showed similar behavior. In particular, the hydraulic part behaved almost identically.

For the time-averaged values, $\bar{f}_{r}$ of $\beta_{b 2}=8$ was located near the origin at each part for $\varphi=0.019-0.030$, whereas $\bar{f}_{r}$ of $\beta_{b 2}=24$ behaved similarly for $\varphi=0.030$. At these flow rates, $\bar{f}_{r}$ was minimized at each part and increased as the flow rate deviated from these points. For $\bar{f}_{r}$ of each part, in every impeller, $\bar{f}_{r}$ was highest for the hydraulic part, followed by the rear shroud and front shroud parts. The value of $\bar{f}_{r}$ at each part of the three impellers was approximately the same. In particular, $\bar{f}_{r}$ was almost identical for $\beta_{b 2}=8$ and 16 in the hydraulic part for the total flow rate domain. This result is due to the pressure component, which will be subsequently discussed.

For the root-mean-square values of the fluctuating component $\Delta f_{r}$ for every impeller, a minimum of $\varphi=0.019$ was set for the total, hydraulic part and rear shroud part. The value increased as the flow rate deviated from the minimum point. For $\varphi=0.008$, the root-mean-square values of $\Delta f_{r}$ were approximately the same for the total, hydraulic part and rear shroud part, whereas the values were larger for higher values of $\beta_{b 2}$ at increased flow rates. This result is presumably due to the increased load on the blade for increased flow rates for impellers with higher $\beta_{b 2}$. In the front shroud part, the root-mean-square value of $\Delta f_{r}$ was highest for $\beta_{b 2}=24$ at $\varphi=0.047$. However, as the flow rate decreased from this point, it fell to below those of $\beta_{b 2}=16$ and 8 at $\varphi=0.019$. This result is believed to have occurred because lower flow rates lead to increased leakage flow rates for impellers with larger $\beta_{b 2}$ [15]. Moreover, for every impeller, the root-meansquare values of $\Delta f_{r}$ for the rear and front shroud parts were smaller than that of the hydraulic part.

From the aforementioned findings, it can be concluded that the hydraulic part is dominant for each impeller regarding time-averaged values and fluctuating component of the total radial thrust. Next, we examine the components of the radial thrust on the hydraulic part as follows.

Figures 9(a) and 9(b) show the component analysis values at their maximum efficiency point of $\varphi=0.030$ for $\beta_{b 2}=8$ and 24 and the Lissajous figures of the above-calculated radial thrust coefficient of the hydraulic part. For both impellers, the component analysis values and the Lissajous figures nearly matched. The same approximate match was noted for other flow rates, verifying the validity of the component analysis.

Figures 10(a)-10(c) and 11(a)-11(c) show the Lissajous figures of the inertia, momentum, and pressure components of the radial thrust of the hydraulic part for $\beta_{b 2}=8$ and 24. Figures 12(a)-12(c) show the time-averaged $\bar{f}_{r}$ and the fluctuating and root-mean-square values of the inertia, momentum, and pressure components for $\beta_{b 2}=8,16$, and 24 . We omitted the time-averaged and fluctuating values of the shear stress component because they were considerably small.

For the time-averaged values $\bar{f}_{r}$, the inertia component of $\bar{f}_{r}$ was near the origin for different flow rates and for 


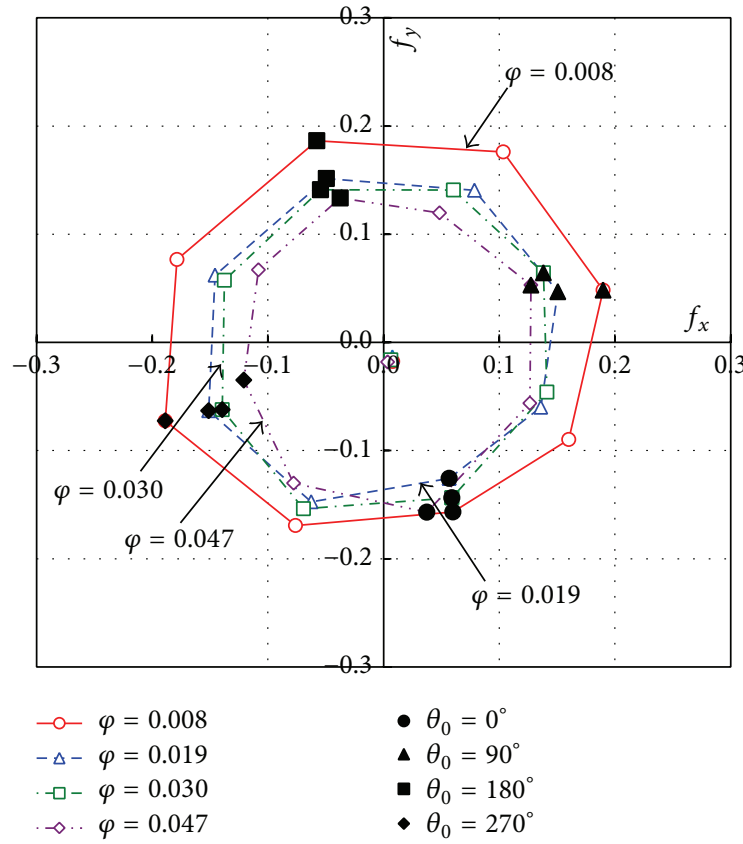

(a) Inertia component

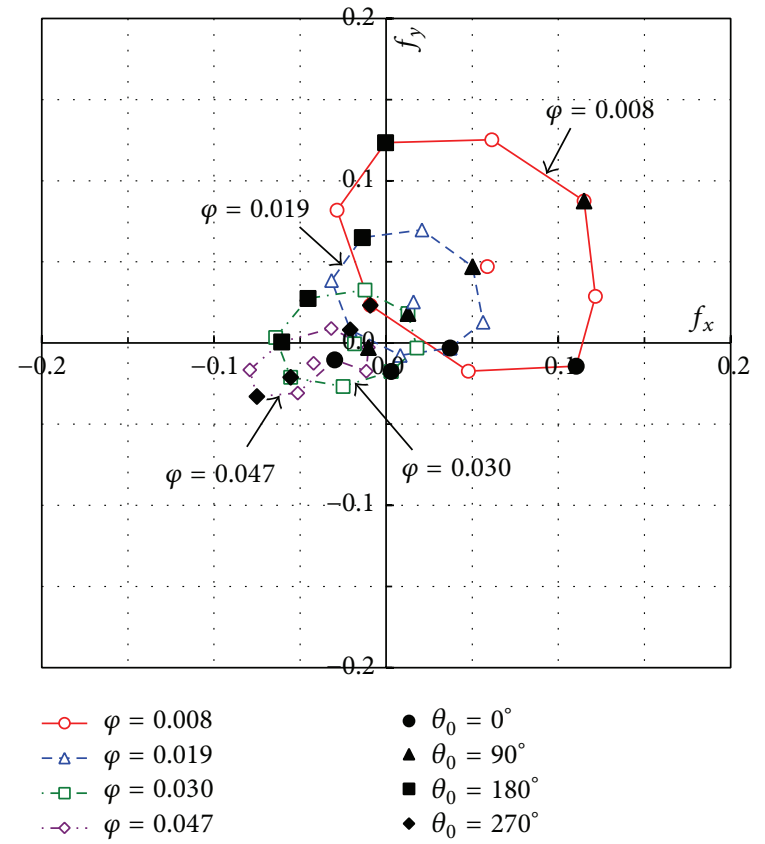

(b) Momentum component

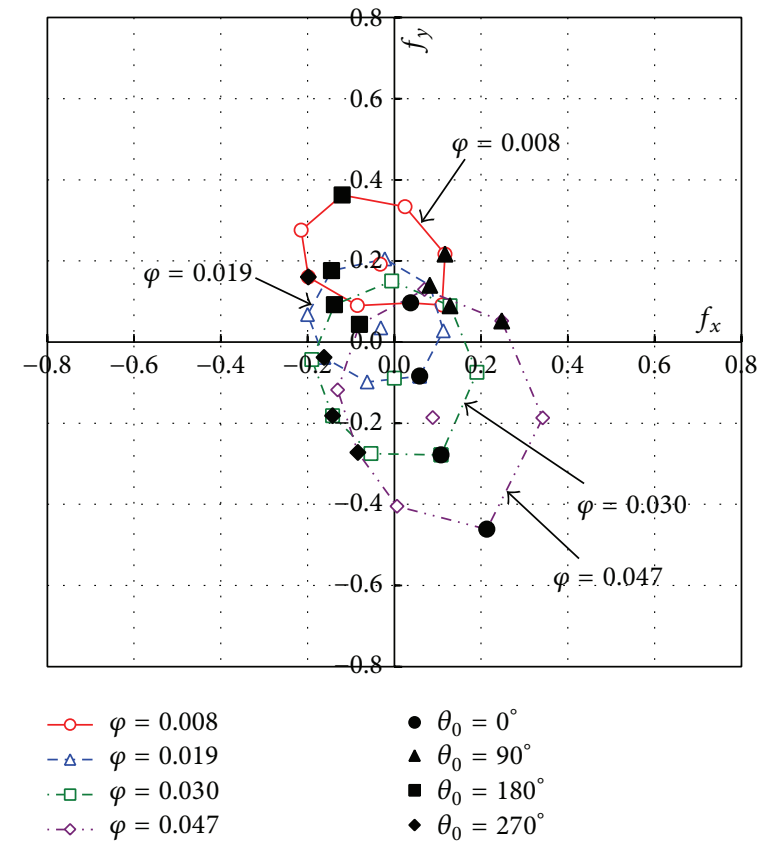

(c) Pressure component

FIGURE 10: Lissajous figures of each component of radial thrust coefficient $\left(\beta_{b 2}=8\right)$.

each impeller. That is, $\bar{f}_{r}$ of the inertia component was small and constant for different flow rates and for each impeller. Moreover, $\bar{f}_{r}$ of the momentum component in each impeller reached its minimum at $\varphi=0.030$ and increased as the flow rate deviated from that point. For points other than $\varphi=0.030$, impellers with higher $\beta_{b 2}$ had higher $\bar{f}_{r}$. In each impeller, $\bar{f}_{r}$ was located in the direction of the beginning of the volute winding for a low flow rate of $\varphi=0.008$ and moved in the $-x$ direction or the direction opposite the outlet, as the flow rate increased. To investigate the behavior of the momentum component of $\bar{f}_{r}$, we determined the absolute velocity vectors at the impeller outlet for $\beta_{b 2}=8$ and 24 in Figures 13(a), 13(b), 14(a), and 14(b), respectively. The blade phase angle was $\theta_{0}=0^{\circ}$. For a low flow rate of $\varphi=0.008$, the stagnation point was located toward the outlet rather than the volute tongue for $\beta_{b 2}=8$ and 24 , indicating a strong recycle flow. 


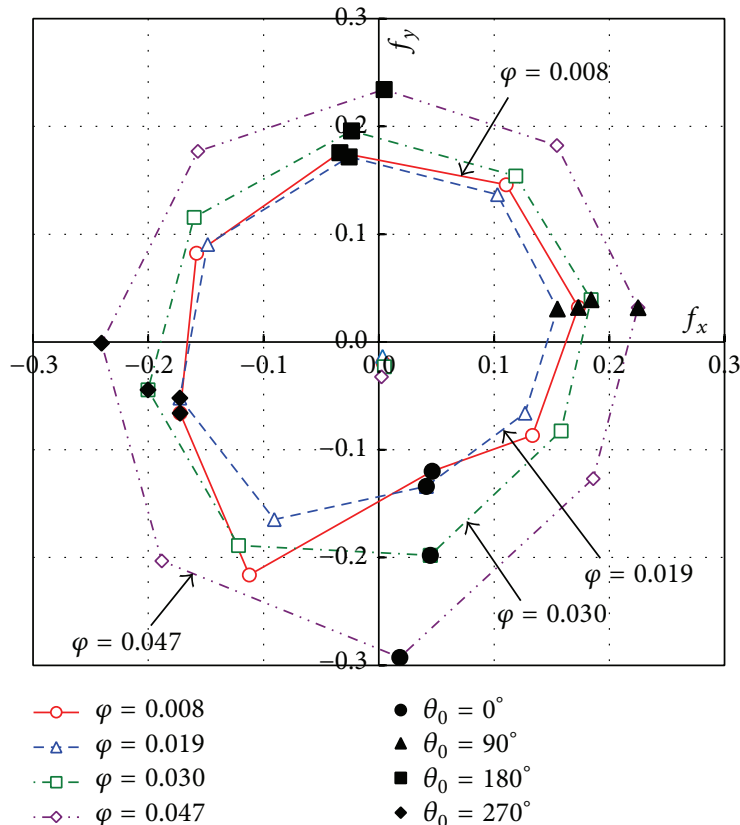

(a) Inertia component

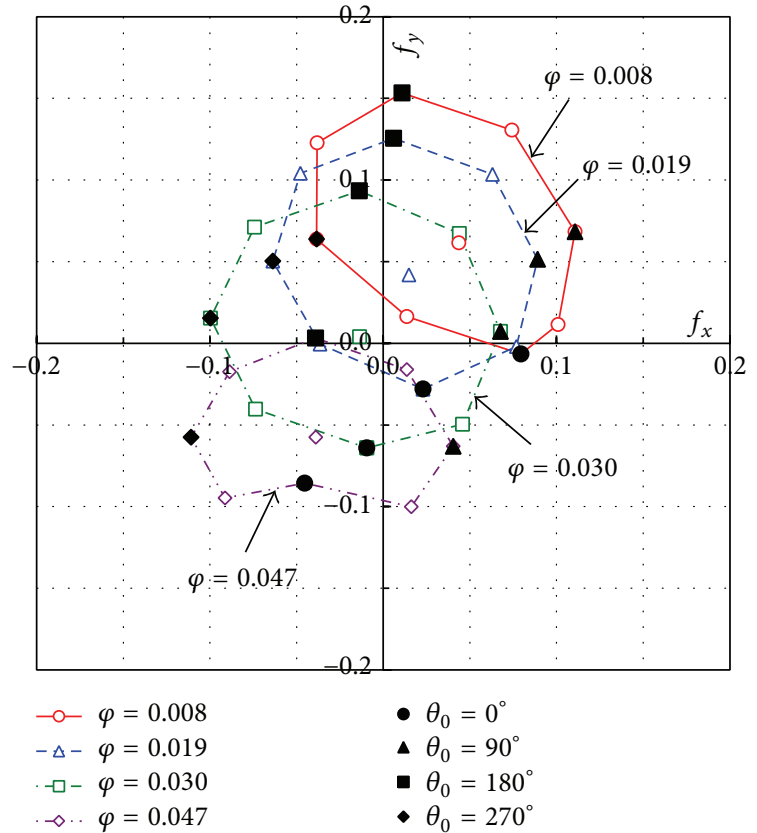

(b) Momentum component

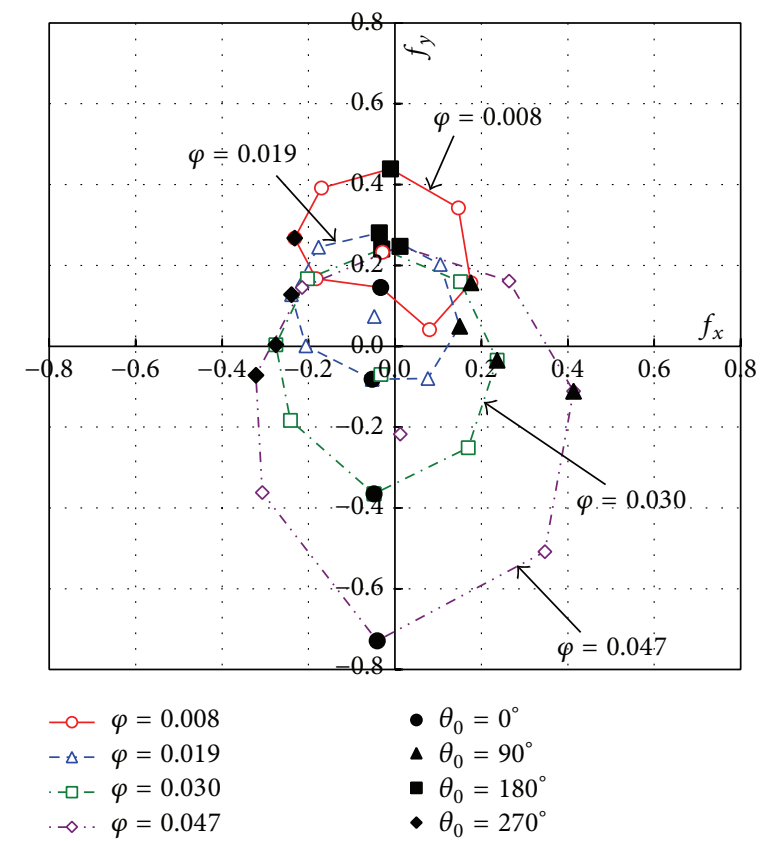

(c) Pressure component

FIGURE 11: Lissajous figures of each component of radial thrust coefficient $\left(\beta_{b 2}=24\right)$.

Therefore, flows from the impeller to the beginning of the volute winding are prevented and flows toward parts other than the beginning of the volute winding become relatively large; moreover, $\bar{f}_{r}$, in turn, presumably moves toward the beginning of the volute winding. For a high flow rate of $\varphi=$ 0.047 , however, the stagnation point for $\beta_{b 2}=8$ was near the beginning of the volute winding due to the high flow rate. The stagnation point for $\beta_{b 2}=24$ was located near the volute tongue but on the side of the winding start of the volute. Therefore, flows from the impeller largely proceeded toward the outlet, including the beginning of the volute winding. The movement of $\bar{f}_{r}$ in the $-x$ direction, or opposite the outlet, may have been due to the counteraction to this phenomenon. Similar to the radial thrust in the hydraulic part shown in Figures 6(b) and 7(b), $\bar{f}_{r}$ of the pressure component in every impeller was located near the origin for $\varphi=0.019-0.030$ and 


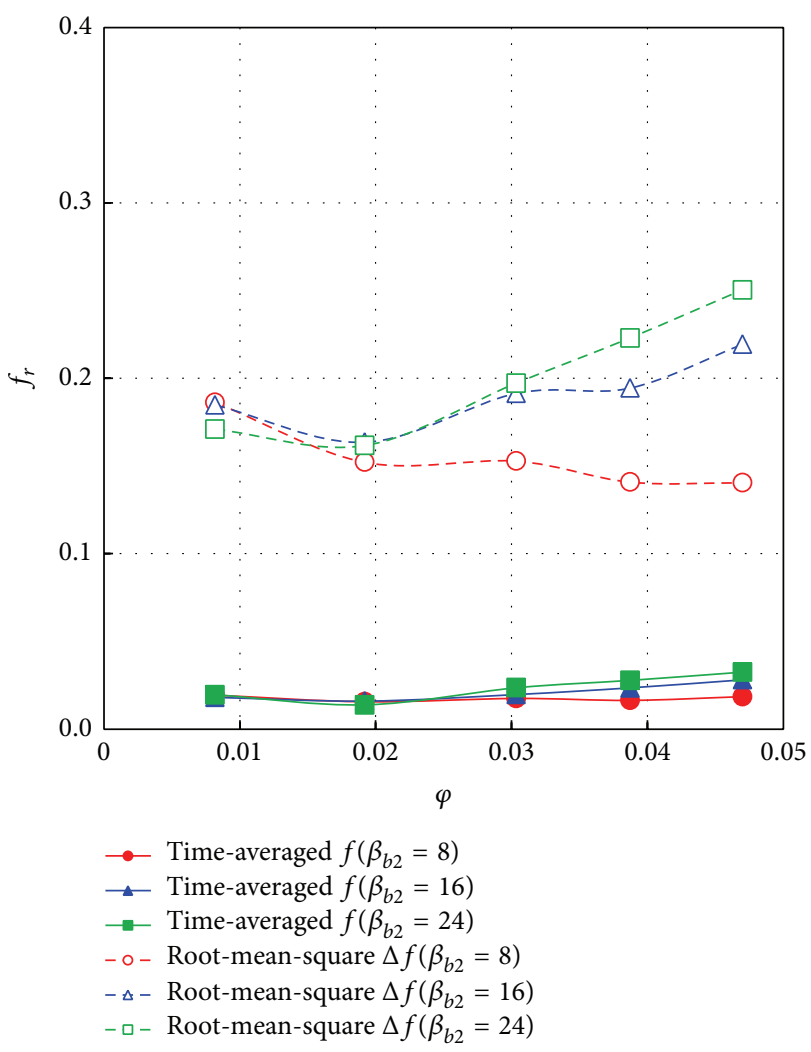

(a) Inertia component

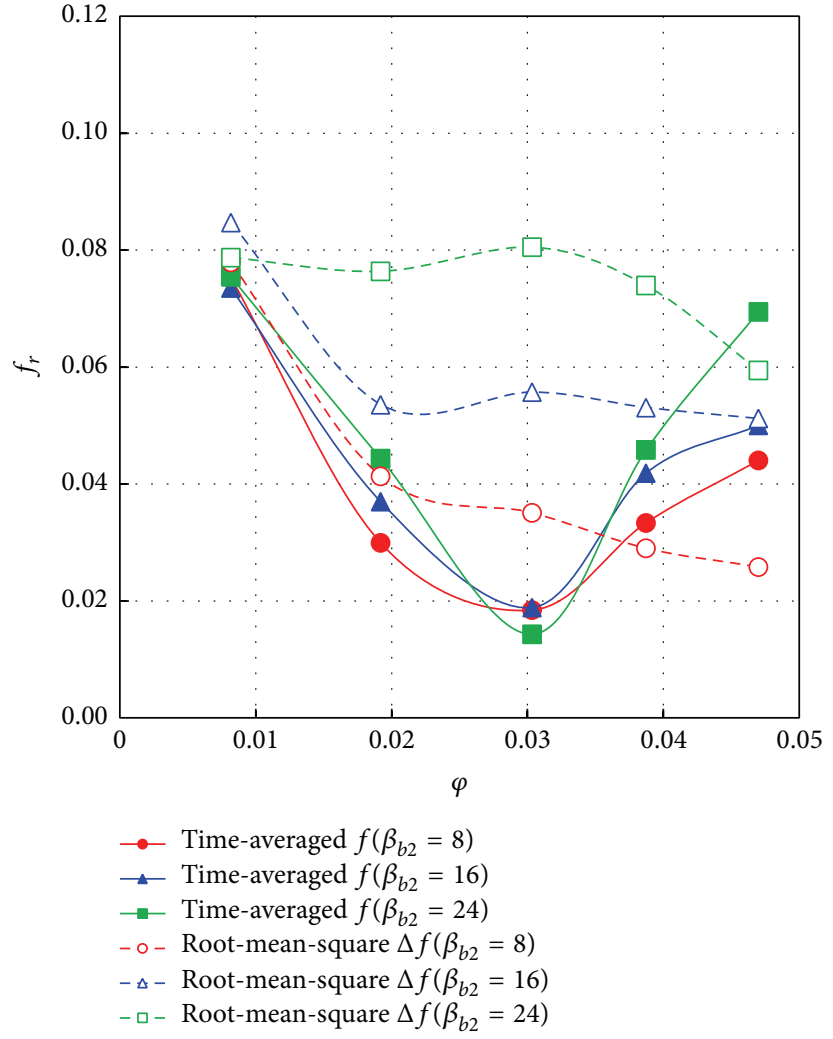

(b) Momentum component

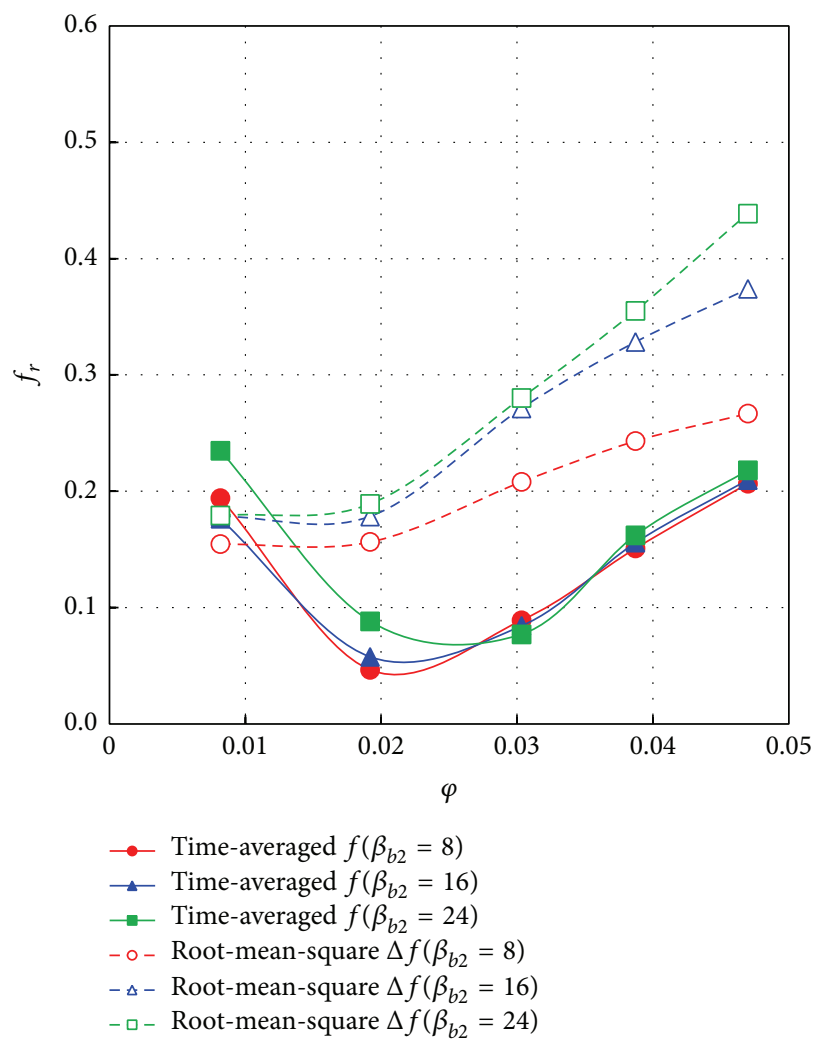

(c) Pressure component

FIGURE 12: Each component of radial thrust coefficient $\left(f_{r}\right)$ as functions of flow rate coefficient $(\varphi)$. 


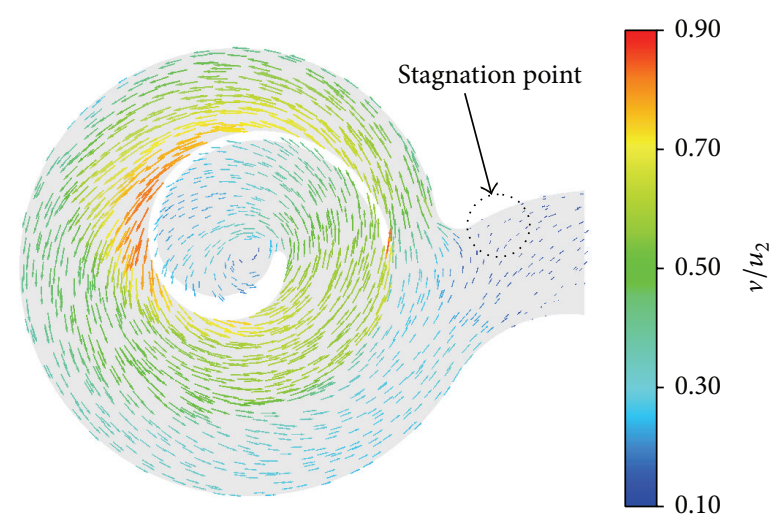

(a) $\varphi=0.008$

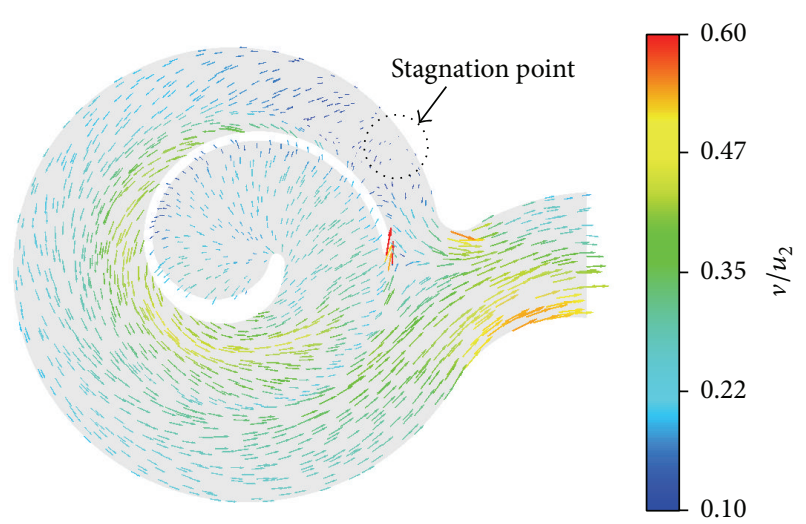

(b) $\varphi=0.047$

FIGURE 13: Absolute velocity vectors $\left(\beta_{b 2}=8, \theta_{0}=0^{\circ}\right)$.

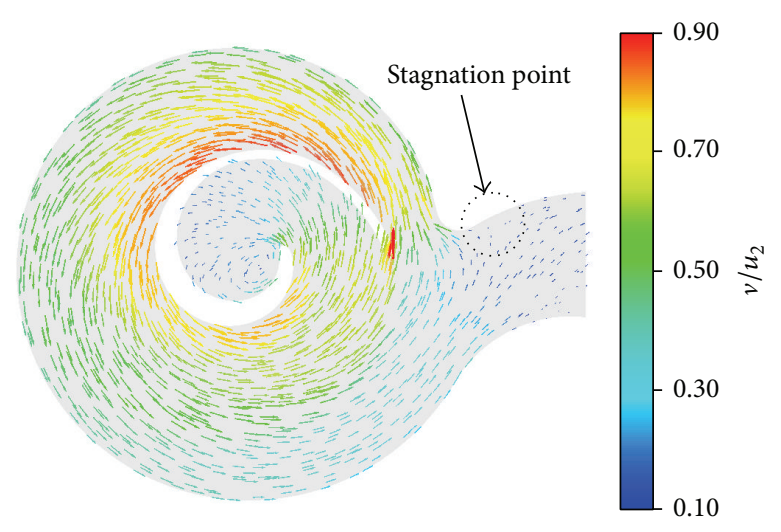

(a) $\varphi=0.008$

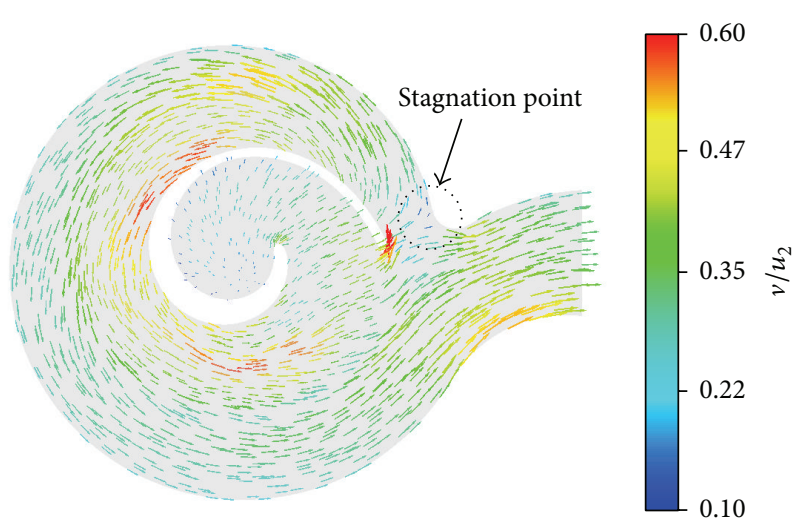

(b) $\varphi=0.047$

FIGURE 14: Absolute velocity vectors $\left(\beta_{b 2}=24, \theta_{0}=0^{\circ}\right)$.

moved in the $+y$ direction for decreased flow rates and in the $-y$ direction for increased flow rates. That is, $\bar{f}_{r}$ of the pressure component in each impeller achieved its minimum at $\varphi=0.019-0.030$ and increased as the flow rate deviated from that point. Therefore, the pressure component of the time-averaged value of the radial thrust in the hydraulic part was dominant for each impeller. Figure 15 shows the circumferential direction distribution of the time-averaged pressure coefficient $C_{P}$ at the monitoring point in the impeller outlet shown in Figure 2. Here, the pressure coefficient $C_{P}$ is the time-averaged value for a single rotation of the runner. At $\varphi=0.030, C_{P}$ was nearly constant for each circumferential direction in each impeller. The value of $C_{P}$ was high near the winding end of the volute at $\varphi=0.008$ and near the beginning of the volute winding at $\varphi=0.047$, which corresponds to the behavior of $\bar{f}_{r}$ of the pressure component. The value of $\bar{f}_{r}$ of the pressure component was low for each $\beta_{b 2}$ and nearly the same likely because $C_{P}$ was higher for impellers with higher $\beta_{b 2}$ at any flow rate but increased uniformly in each circumferential direction (Figure 15). It has been confirmed that the calculated values of $C_{P}$ at the impeller outlet matched well the experimental values [14].
For the root-mean-square values of the fluctuating component $\Delta f_{r}$, the $\Delta f_{r}$ of the inertia component in each impeller with the same blade phase angle $\theta_{0}$ was in a similar direction for different flow rates. The root-mean-square value of $\Delta f_{r}$ of the inertia component for $\beta_{b 2}=8$ decreased with increasing flow rates. In contrast, it achieved its minimum at $\varphi=$ 0.019 for $\beta_{b 2}=16$ and 24 and increased as the flow rate deviated from that point. The value of $\Delta f_{r}$ was nearly the same for all impellers at values of $\varphi=0.019$ and below; for $\varphi=0.019$ and above, however, impellers with higher $\beta_{b 2}$ had higher values. The root-mean-square values of the momentum component were small compared to those of other components; however, they increased for $\beta_{b 2}=8$ and 16 when the flow rate decreased. Impellers with higher $\beta_{b 2}$ had higher values, which can likely be attributed to the increased circumferential component $v_{u 2}$ of the absolute velocity of the blade outlet for higher $\beta_{b 2}$. For every impeller, the rootmean-square value of $\Delta f_{r}$ of the pressure component for $\varphi=$ 0.019 increased with an increase in flow rate. At $\varphi=0.008$, the root-mean-square values $\Delta f_{r}$ of the pressure and inertia components were similar, although the pressure component was higher than the other components for any other flow 


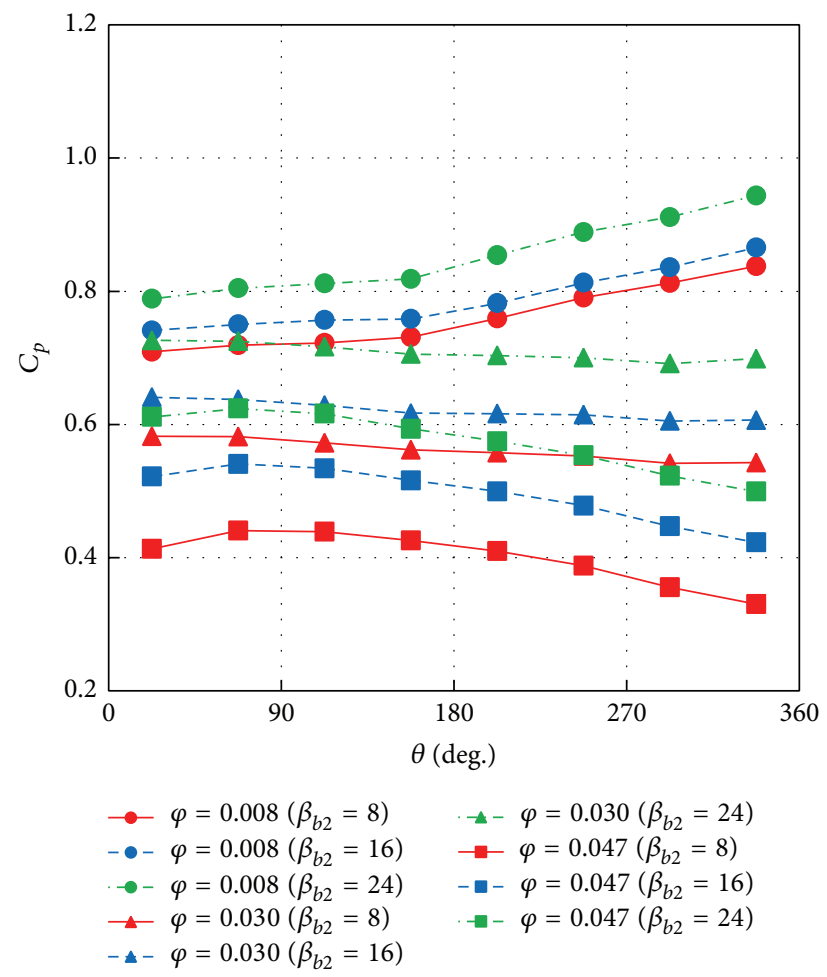

FIGURE 15: Circumferential direction distributions of time-averaged pressure coefficient $\left(C_{P}\right)$.

rate. Therefore, the pressure component of the fluctuating component of the radial thrust in the hydraulic part was dominant for each impeller. The value of $\Delta f_{r}$ was nearly the same for any impeller for $\varphi=0.008$ and below. For $\varphi=0.019$ and above, however, impellers with higher $\beta_{b 2}$ had higher values, similar to the radial thrust on the hydraulic part. This result is likely due to the larger load on the blade for higher $\beta_{b 2}$ for the increased flow rates and increased pressure distribution around the impeller.

On the basis of these findings, it can be concluded that, for low flow rates, each component has a similar magnitude for every impeller. Moreover, the radial thrust on the hydraulic part is nearly the same magnitude. For increased flow rates, the time-averaged values of the pressure component were similar for all impellers, although the fluctuating component was larger for impellers with larger blade outlet angles. Moreover, the fluctuating inertia component of $\beta_{b 2}=8$ decreased as the flow rate increased, whereas those of $\beta_{b 2}=$ 16 and 24 increased. Therefore, the radial thrust on the hydraulic part is significantly higher for impellers with high blade outlet angles.

5.3. Axial Thrust and Comparisons of Its Components. The time-averaged values of the axial thrust coefficients for the total, hydraulic part, rear shroud part, and front shroud part for each impeller are shown in Figures 16(a)-16(d). Here, each axial thrust was obtained by integrating the pressure and the shear stress on the wall of each impeller part.

The absolute value of the total axial thrust coefficient increased with decreasing flow rates, and the impeller with $\beta_{b 2}=24$ had the highest values. No significant differences were noted between $\beta_{b 2}=8$ and 16; $\beta_{b 2}=8$ had a higher value for low flow rates. For every impeller, the axial thrust coefficient was highest in the $-z$ direction. The axial thrust coefficient on the front shroud part was applied in the $+z$ direction and was slightly smaller than that on rear shroud part. The axial thrust coefficient on the hydraulic part was also applied in the $+z$ direction but was smaller in magnitude. The axial thrust coefficients for the rear and front shroud parts were higher for larger $\beta_{b 2}$ values; moreover, that for the hydraulic part did not change significantly for different $\beta_{b 2}$ values. However, unlike the total axial thrust coefficient, that of the hydraulic part increased with an increase in flow rate, comprising $25.5 \%$ of the total axial thrust coefficient at $\varphi=$ 0.047 for $\beta_{b 2}=8$. Subsequently, we examine the components of the axial thrust on the hydraulic part.

Figure 17 shows the component analysis values of the time-averaged values of the axial thrust for the hydraulic part for $\beta_{b 2}=8$ and 24 . For both impellers, the component analysis values and the calculated values nearly matched, which validates the analysis results.

Figures 18(a)-18(c) show the time-averaged values of the inertia, momentum, and pressure components for the hydraulic part for $\beta_{b 2}=8,16$, and 24 . We omitted the shear stress component because it was quite small.

A comparison of each component revealed that the inertia component was relatively small for $\beta_{b 2}$ values other than $16^{\circ}$. For every impeller, the momentum component was in the $+z$ direction. It was the largest for flow rates other than $\varphi=0.008$ and increased with increasing flow rates. 


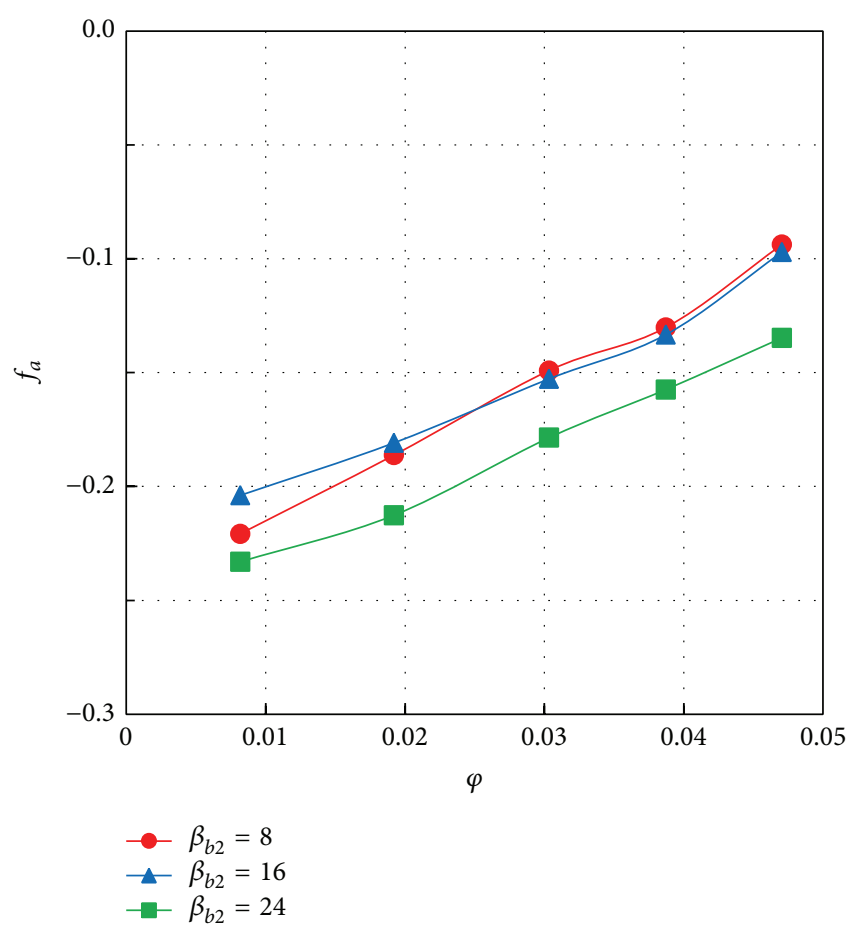

(a) Total

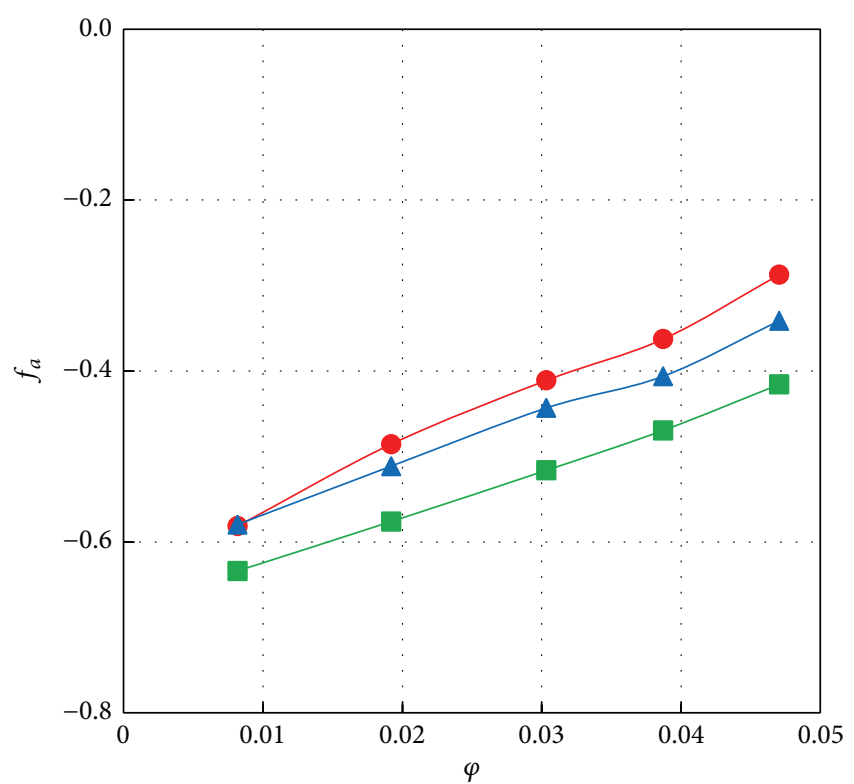

$-\beta_{b 2}=8$
$-\beta_{b 2}=16$
$-\beta_{b 2}=24$

(c) Rear shroud part

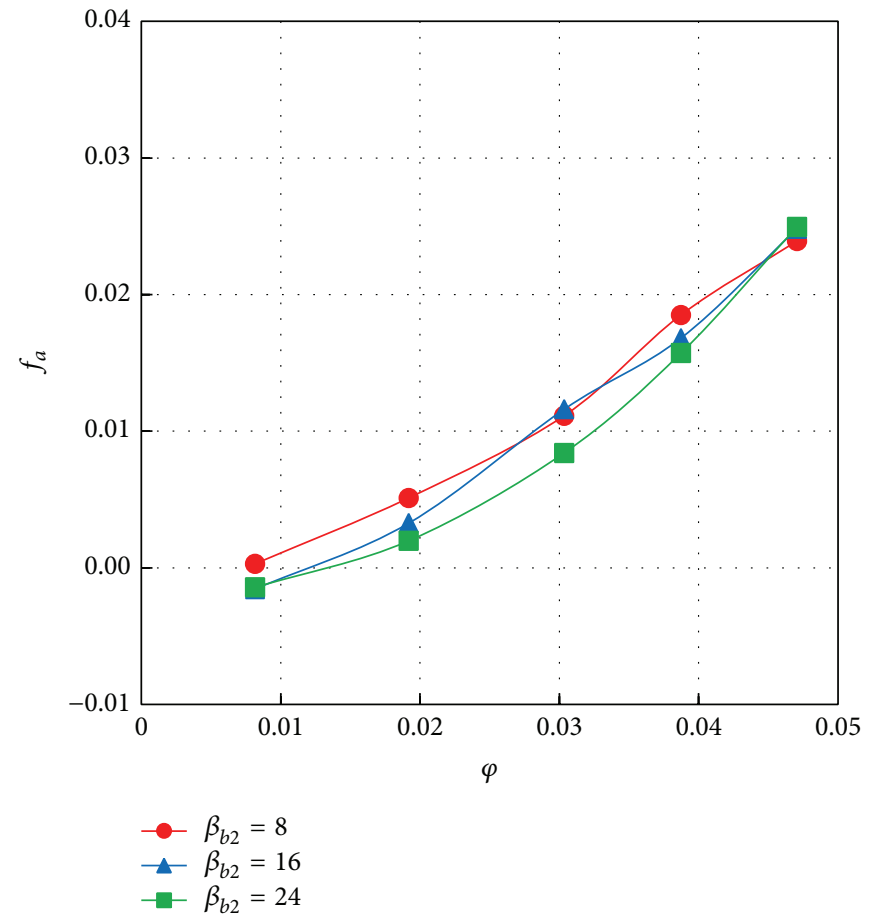

(b) Hydraulic part

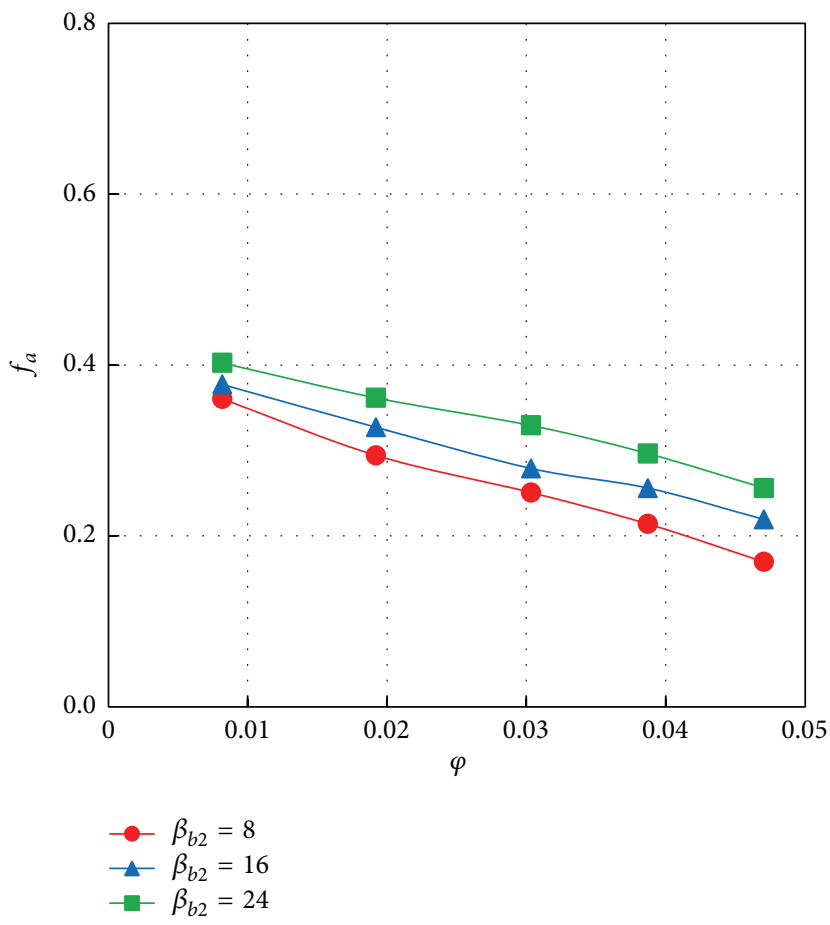

(d) Front shroud part

FIGURE 16: Time-averaged values of axial thrust coefficient $\left(f_{a}\right)$ on each part as functions of flow rate coefficient $(\varphi)$.

Flow into the impeller shifted from the axial direction to the radial direction and received a counteracting force in the $+z$ direction. The momentum component was the force on the impeller; the momentum increased with an increase in flow rate, thus increasing the momentum component. For every impeller, the pressure component was in the $-z$ direction, and its absolute value increased with an increase in flow rate. Therefore, for each impeller, the momentum component of the time-averaged values of the axial thrust on the hydraulic part was dominant and was applied in the $+z$ direction. For 


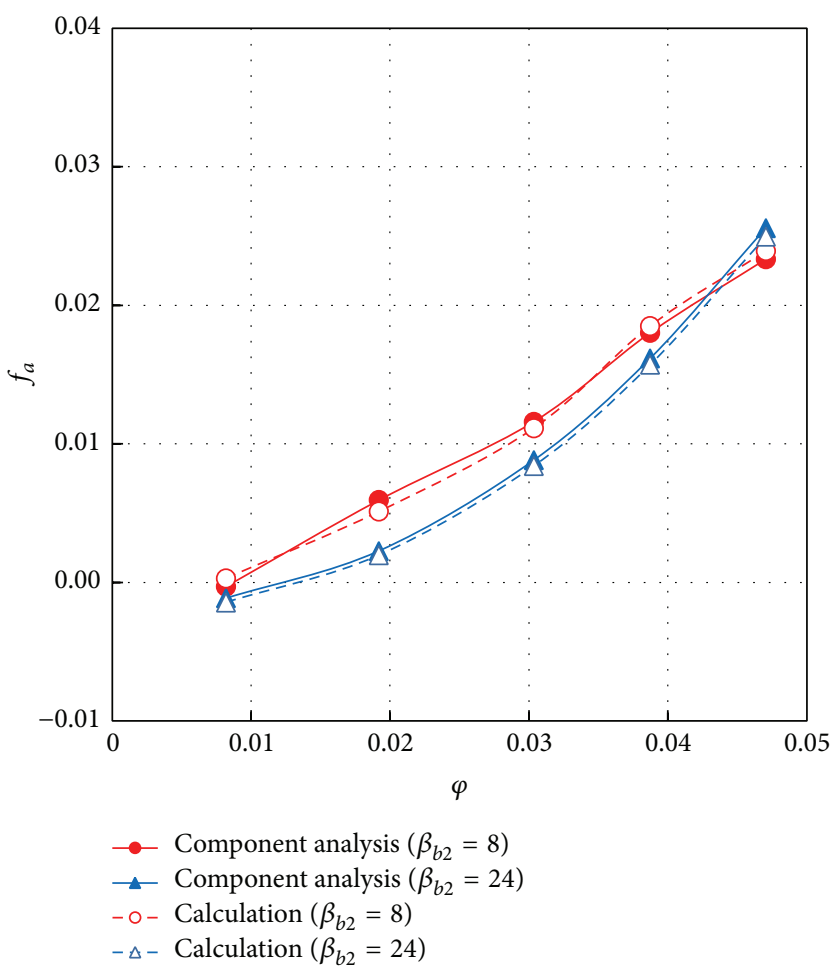

FIGURE 17: Time-averaged values of hydraulic part axial thrust coefficient $\left(f_{a}\right)$ as functions of flow rate coefficient $(\varphi)$.

higher flow rates, however, more than half of the momentum components were counteracted by the pressure components.

\section{Conclusions}

We studied the effects of blade outlet angles of closed, singleblade centrifugal pumps on radial and axial thrusts and their components. The results are presented in the following points:

(1) The pressure component of the time-averaged value and fluctuating component of the radial thrust in the hydraulic part were dominant for each impeller.

(2) For small flow rates each component had similar magnitudes for every impeller, and the radial thrust on the hydraulic part was approximately the same magnitude. For increased flow rates, the time-averaged values of the pressure component were similar for all impellers, although its fluctuating component was higher for impellers with larger blade outlet angles. Moreover, the fluctuating inertia component of the impeller with a blade outlet angle of $8^{\circ}$ decreased as the flow rate increased, whereas those with $16^{\circ}$ and $24^{\circ}$ angles increased. Therefore, the radial thrust on the hydraulic part was significantly higher for impellers with high blade outlet angles.

(3) For each impeller, the momentum component of the time-averaged values of the axial thrust on the hydraulic part was dominant and was applied in the $+z$ direction. For higher flow rates, however, more than half of the momentum components were counteracted by the pressure components.

\section{Nomenclature}

A: Area $^{2}$

$b$ : $\quad$ Blade width $\mathrm{m}$

$C_{P}:$ Pressure coefficient $=\left(P-P_{s}\right) /\left(\rho u_{2}^{2} / 2\right)$

$D$ : Impeller diameter $m$

$f_{a}:$ Axial thrust coefficient $=F_{a} /\left(\rho u_{2}^{2} \pi r_{2}^{2} / 2\right)$

$f_{r}:$ Radial thrust coefficient $=F_{r} /\left(\rho u_{2}^{2} b_{2} r_{2} / 2\right)$

$F_{a}$ : Axial thrust $\mathrm{N}$

$F_{r}$ : Radial thrust $\mathrm{N}$

$g$ : Gravitational acceleration $\mathrm{m} / \mathrm{s}^{2}$

$H$ : Total head $\mathrm{m}$

$L$ : Shaft power $\mathrm{W}$

$n: \quad$ Rotational speed $\mathrm{min}^{-1}$

$P$ : Static pressure (gauge pressure) $\mathrm{Pa}$

Q: Flow rate $\mathrm{m}^{3} / \mathrm{s}$

$r$ : Impeller radius $\mathrm{m}$

$u$ : Circumferential velocity $\mathrm{m} / \mathrm{s}$

$v$ : Absolute velocity $\mathrm{m} / \mathrm{s}$

$\beta_{b}$ : Blade angle ${ }^{\circ}$.

\section{Greek Letters}

$\eta: \quad$ Pump efficiency $=\rho g Q H / L$

$\theta$ : Circumferential angle ${ }^{\circ}$

$\theta_{0}$ : Blade phase angle ${ }^{\circ}$

$\rho$ : Fluid density $\mathrm{kg} / \mathrm{m}^{3}$ 

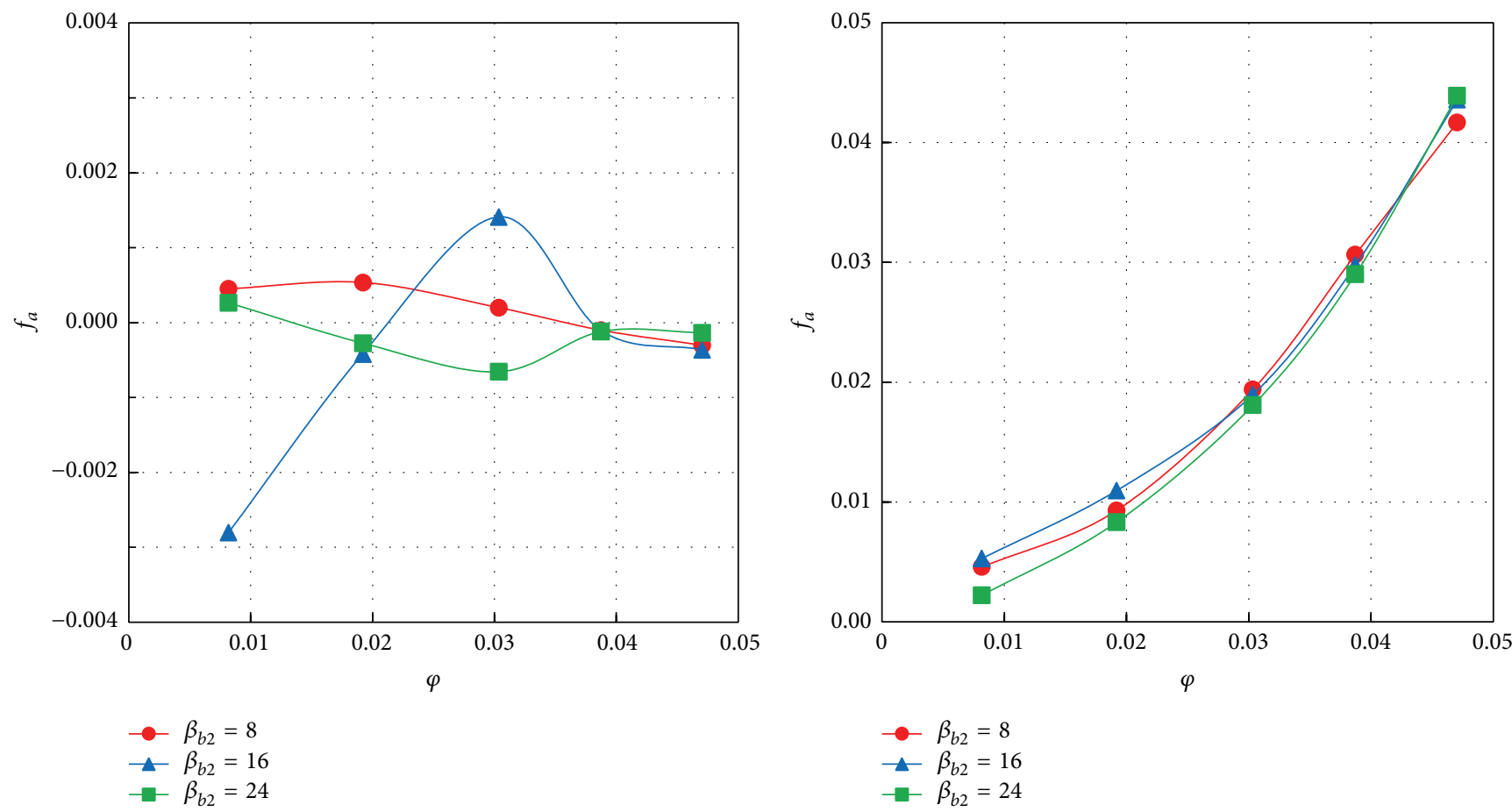

(a) Inertia component

(b) Momentum component

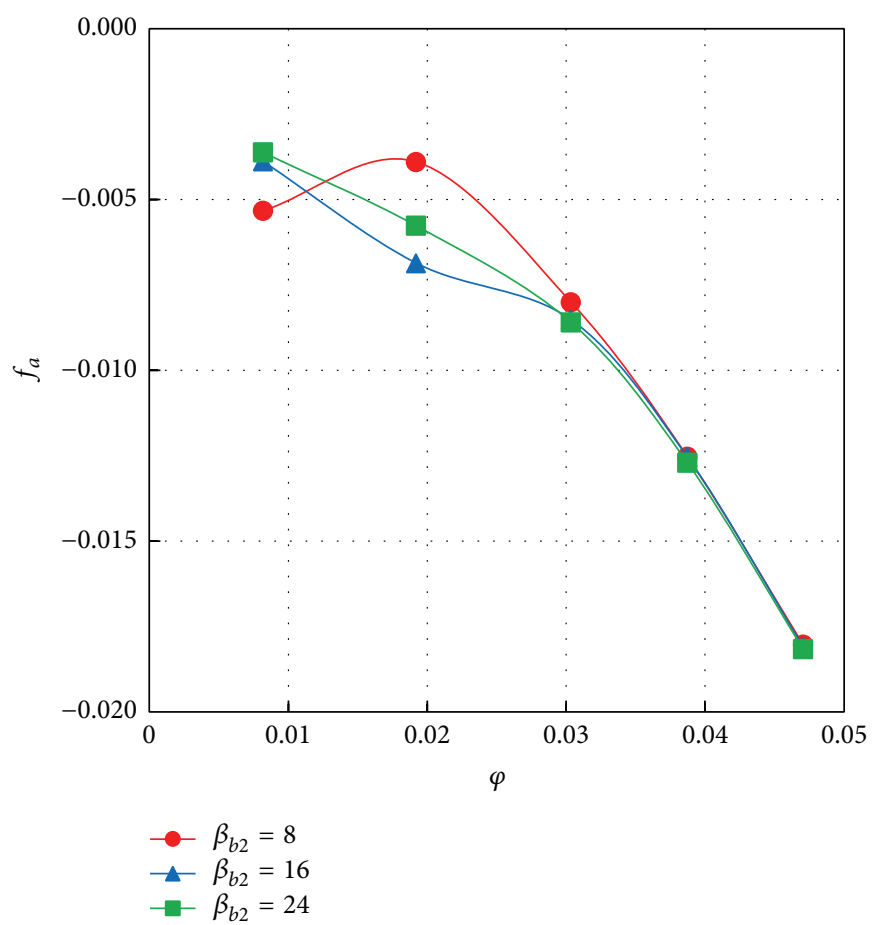

(c) Pressure component

FIGURE 18: Time-averaged values of each component of axial thrust coefficient $\left(f_{a}\right)$ as functions of flow rate coefficient $(\varphi)$. 
$\varphi:$ Flow rate coefficient $=Q /\left(\pi D_{2} b_{2} u_{2}\right)$

$\psi$ : Head coefficient $=H /\left(u_{2}^{2} / 2 g\right)$.

\section{Subscripts}

0: Impeller suction inlet

1: Blade inlet

2: Blade outlet

a: Axial component

$r$ : Radial component

$u$ : Circumferential component

$s$ : Suction side reference position

$x: x$ component

$y: y$ component

$z: z$ component.

\section{Conflict of Interests}

The authors declare that there is no conflict of interests regarding the publication of this paper.

\section{Acknowledgment}

This work was supported by JSPS KAKENHI Grant no. 24760130.

\section{References}

[1] Japan Institute of Wastewater Engineering Technology, Wastewater Manhole Pump Technology Manual-June 1997, 1997.

[2] Y. Yoshida, T. Sakatani, T. Kawakami, and Y. Tsujimoto, "Study of hydraulic unbalance caused by geometrical manufacturing errors of centrifugal impeller (1st report: experimental results)," Turbomachinery, vol. 23, no. 2, pp. 72-77, 1995.

[3] T. Okamura, "Radial thrust in centrifugal pumps with single vane impeller," Transactions of the Japan Society of Mechanical Engineers, Series B, vol. 45, no. 398, pp. 1458-1464, 1979.

[4] M. Aoki, "Instantaneous inter-blade pressure distributions and fluctuating radial thrust in a single-blade centrifugal pump," Transactions of the Japan Society of Mechanical Engineers, Series $B$, vol. 50, no. 451, pp. 661-669, 1984.

[5] F.-K. Benra, H. J. Dohmen, and O. Schneider, "Calculation of hydrodynamic forces and flow induced vibrations of centrifugal sewage water pumps," in Proceedings of the 4th ASME/JSME Joint Fluids Engineering Conference, Paper FEDSM2003-45102, pp. 603-608, Honolulu, Hawaii, USA, July 2003.

[6] F.-K. Benra, H. J. Dohmen, and M. Sommer, "Experimental investigation of hydrodynamic forces for different configurations of single-blade centrifugal pumps," in Proceedings of the 11th International Symposium on Transport Phenomena and Dynamics of Rotating Machinery (ISROMAC '06), Honolulu, Hawaii, USA, 2006.

[7] I. Ishida, "The consideration in the radial thrust decrease of centrifugal pump," Turbomachinery, vol. 30, no. 12, pp. 741-746, 2002.

[8] F.-K. Benra, "Numerical and experimental investigation on the flow induced oscillations of a single-blade pump impeller," Journal of Fluids Engineering, vol. 128, no. 4, pp. 783-793, 2006.

[9] F.-K. Benra, "Application of fluid/structure interaction methods to determine the impeller orbit curves of a centrifugal pump," in
Proceedings of the 5th IASME/WSEAS International Conference on Fluid Mechanics and Aerodynamics, Athens, Greece, 2007.

[10] J. Pei, H. J. Dohmen, S. Q. Yuan, and F.-K. Benra, "Investigation of unsteady flow-induced impeller oscillations of a singleblade pump under off-design conditions," Journal of Fluids and Structures, vol. 35, pp. 89-104, 2012.

[11] Y. Nishi, R. Fujiwara, and J. Fukutomi, "Design method for single-blade centrifugal pump impeller," Journal of Fluid Science and Technology, vol. 4, no. 3, pp. 786-800, 2009.

[12] Y. Nishi, J. Fukutomi, and R. Fujiwara, "Radial thrust of single-blade centrifugal pump," International Journal of Fluid Machinery and Systems, vol. 4, no. 4, pp. 387-395, 2011.

[13] Y. Nishi, J. Fukutomi, and R. Fujiwara, "Effect of blade outlet angle on radial thrust of single-blade centrifugal pump," in Proceedings of the 26th IAHR Symposium on Hydraulic Machinery and Systems, Beijing, China, August 2012.

[14] Y. Nishi, J. Fukutomi, and M. Sakai, "Component analysis of unsteady hydrodynamic force of closed-type centrifugal impeller with single-blade," Transactions of the Japan Society of Mechanical Engineers Series B, vol. 78, no. 796, pp. 2062-2075, 2012.

[15] Y. Nishi and J. Fukutomi, "Effect of blade outlet angle on unsteady hydrodynamic force of closed-type centrifugal pump with single blade," International Journal of Rotating Machinery, vol. 2014, Article ID 838627, 16 pages, 2014.

[16] ANSYS, ANSYS CFX-Solver Theoretical Guide, ANSYS, 2010.

[17] ANSYS Inc, ANSYS CFX-Solver Modeling Guide, 2010.

[18] R. C. Worster, "The flow in volutes and its effect on centrifugal pump performance," Institution of Mechanical Engineers, vol. 177, no. 31, pp. 843-875, 1963.

[19] K. Tanaka, M. Arai, S. Ikeo, and Y. Matsumoto, "Radial thrust in screw centrifugal pump impeller," Turbomachinery, vol. 17, no. 4, pp. 215-221, 1989. 

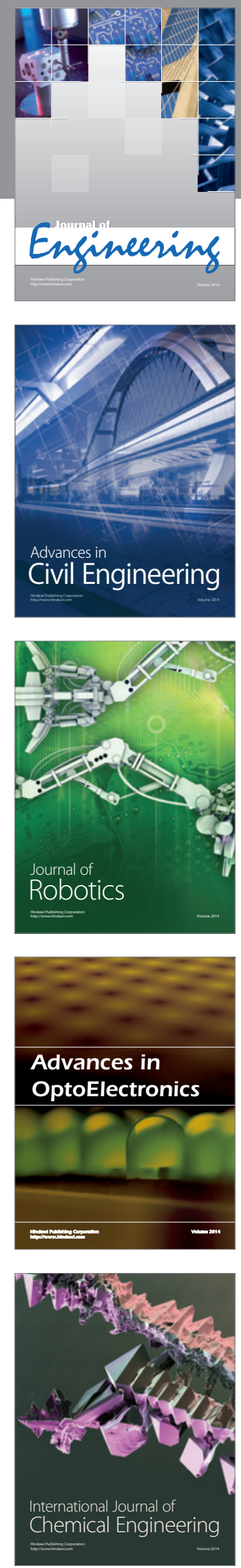

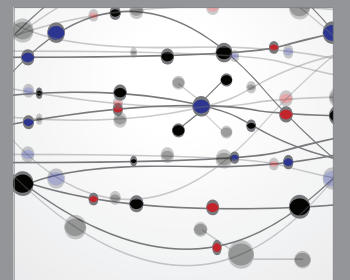

The Scientific World Journal
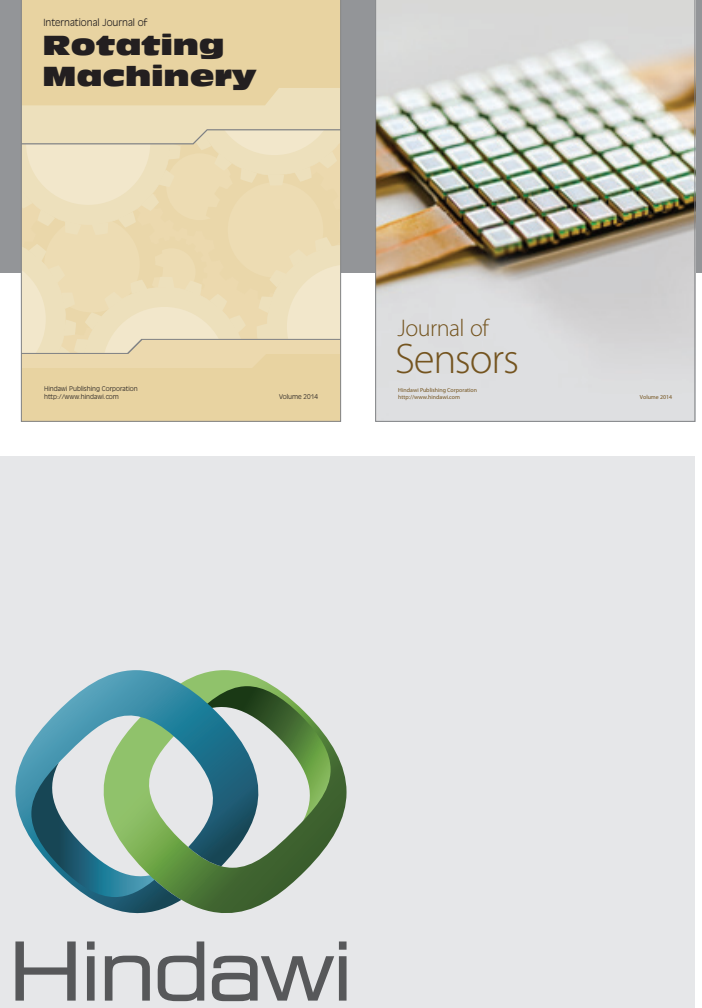

Submit your manuscripts at http://www.hindawi.com
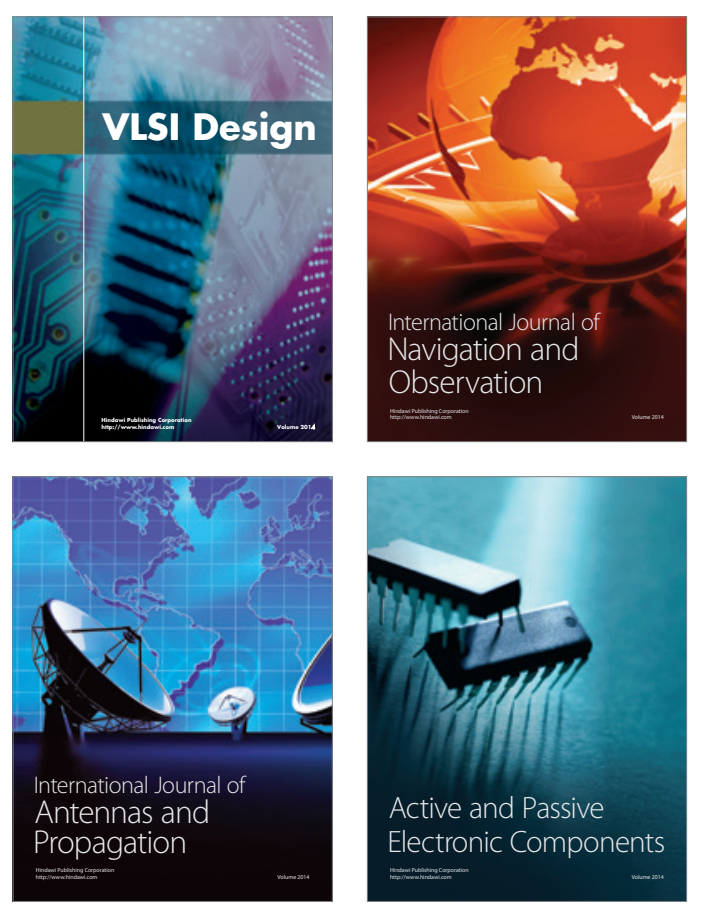
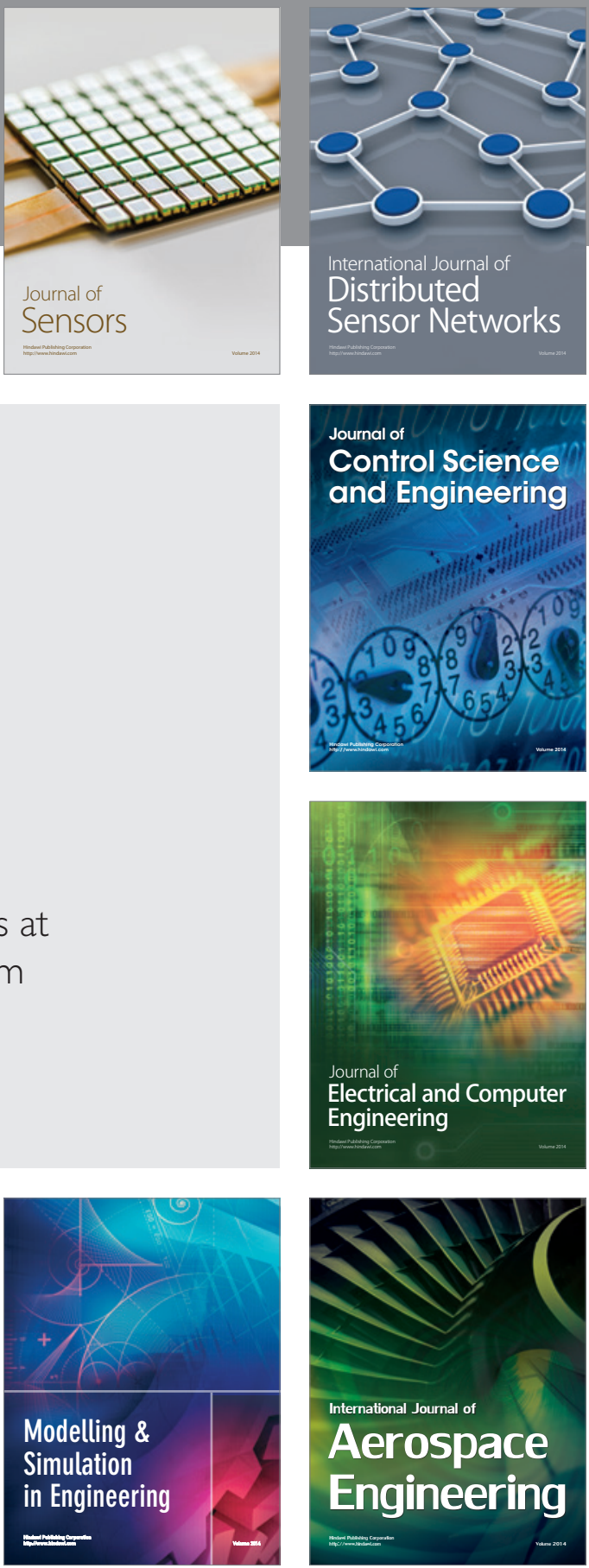

Journal of

Control Science

and Engineering
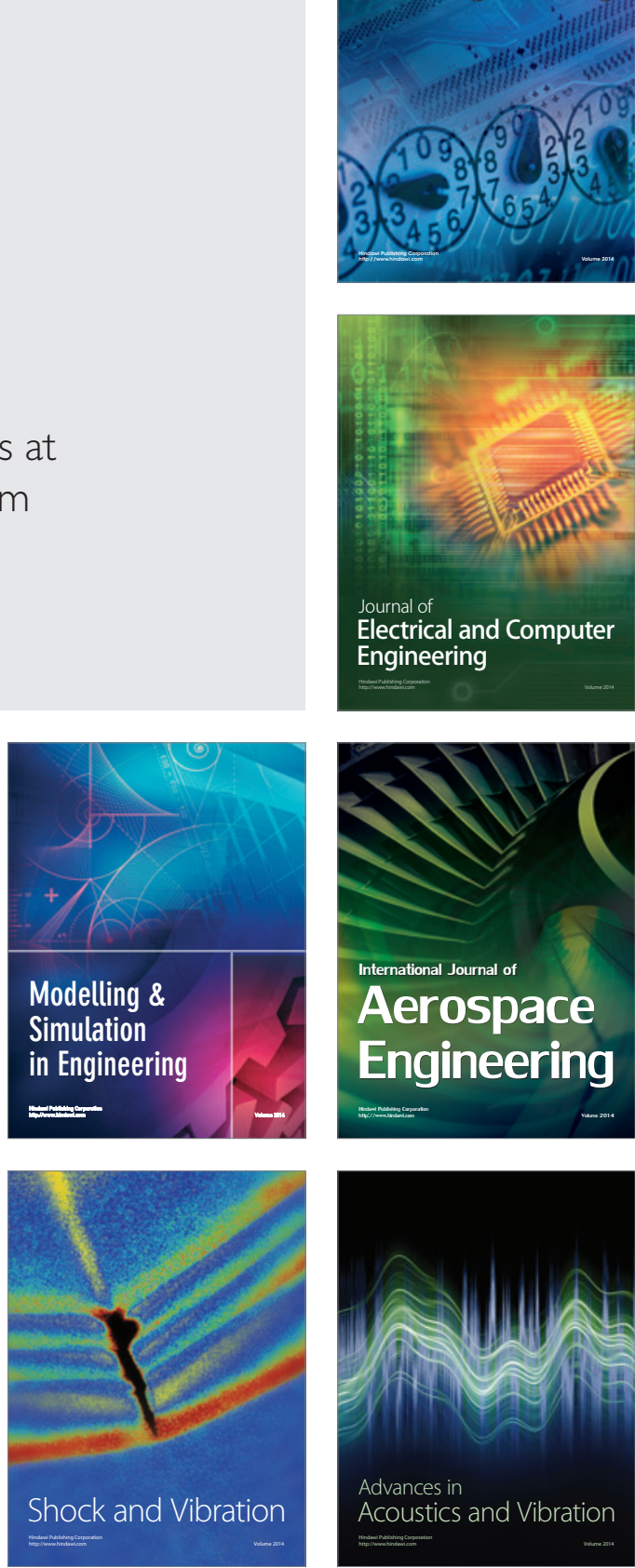